\title{
Distributions and correlations for top quark pair production and decay at the Tevatron and LHC
}

\author{
Werner Bernreuther ${ }^{\alpha}$ and Zong-Guo Si 计 $^{*}$
}

\author{
${ }^{a}$ Institut für Theoretische Physik, RWTH Aachen University, 52056 Aachen, \\ Germany \\ ${ }^{b}$ Department of Physics, Shandong University, Jinan, Shandong 250100, China
}

\begin{abstract}
We investigate a number of observables that are and will be instrumental in the exploration of $t \bar{t}$ production and decay at the Tevatron and the LHC. For this analysis we made a computer program that incorporates besides the NLO QCD corrections to $t \bar{t}$ production and decay also mixed weak-QCD corrections to the production amplitudes, and that allows for studies of correlated versus uncorrelated $t \bar{t}$ events. In this paper we analyze and compute observables mostly for dileptonic $t \bar{t}$ final states to next-toleading order in the strong and weak gauge couplings (NLOW), with selection cuts. We calculate charge asymmetries of the top quark and of $\ell=e, \mu$ and we compare, where possible, with experimental results. We show that top-quark spin correlations affect a leptonic pair asymmetry (which has not been measured so far) by $\sim 7 \%$. We determine several dileptonic angular correlations, which reflect $t \bar{t}$ spin correlations, namely the beam, off-diagonal and helicity correlation, and the opening angle distribution (defined in the $t, \bar{t}$ rest frames) when selection cuts are applied. Our NLOW predictions for the beam, off-diagonal, and helicity correlation for the Tevatron agree with recent measurements by the CDF and D0 experiments. In addition we make predictions for estimators of these correlations as functions of $M_{t \bar{t}}$. These estimators may prove useful for the Tevatron and also in the early rounds of LHC data-analyses, where the event numbers will not be abundant. Furthermore, we compute to NLOW in the gauge couplings two dilepton angular correlations that are defined in the laboratory frame, for correlated and uncorrelated $t \bar{t}$ events at the LHC. Finally, based on our SM results, we identify several observables that allow to search for non-standard parity- and CP-violating interactions, especially with future LHC data.
\end{abstract}

PACS number(s): 12.38.Bx, 13.88.+e, 14.65.Ha

Keywords: hadron collider physics, top quarks, QCD and weak corrections

\footnotetext{
*breuther@physik.rwth-aachen.de

†zgsi@sdu.edu.cn
} 


\section{Introduction}

Exploration of the production and decay of top-quark pairs $(t \bar{t})$ is and will be among the central physics topics at the Tevatron and at the Large Hadron Collider (LHC). On the theoretical side, the investigation of these processes has reached quite a sophisticated level. The next-toleading order (NLO) QCD corrections to the $t \bar{t}$ cross sections and several top-quark distributions have been known for quite some time from the pioneering work of [1-4] and [5, 6], which was extended by threshold resummation calculations [7-10]. The pair cross sections were recently updated by [15 17]. The NLO QCD corrections including the full dependence of the matrix elements on the top-quark spin degrees of freedom were determined in [11 14. The mixed weakinteraction QCD corrections [18 24 and the photonic corrections 25] to $t \bar{t}$ production and the order $\alpha_{s}^{3}$ nonfactorizable QCD corrections [26] are also known. The computations of the NLO QCD corrections to $t \bar{t}+$ jet, $t \bar{t}+b \bar{b}$, and $t \bar{t}+2$ jets, which are important background processes in the search for (the) Higgs boson(s), were reported in [27,28], in [29 31], and in [32] respectively. Differential distributions for polarized semileptonic and non-leptonic decays of the top quark were determined order $\alpha_{s}$ by [33] and [34], respectively, while its total width is known to order $\alpha_{s}^{2}$ [35, 36]. A number of observables that are instrumental in exploring the $t \bar{t}$ production dynamics were investigated in detail 1 . These include the $W$-boson helicity fractions in $t \rightarrow b W$ at NLO in the gauge couplings [40] and to $\alpha_{s}^{2}$ QCD [41], and the top-quark charge asymmetry at the Tevatron in the context of standard model (SM) interactions [2,4,27,42 46]. This asymmetry was measured by the D0 and CDF experiments 47 49. Top-quark spin effects, in particular $t \bar{t}$ spin correlations were analyzed in [50 64] at LO and in [13, 14] at NLO QCD. Measurements of $t \bar{t}$ spin correlations at the Tevatron were reported in [65 67]. Quite recently there has been an increased activity on theoretical and phenomenological issues, including the first determination of the running mass $\bar{m}_{t}$ of the top quark [68] from $\sigma_{t \bar{t}}^{e x p}$, analytic phase-space integration of the NLO QCD parton cross sections $g g, q \bar{q}, g q \rightarrow t \bar{t} X$ [69], threshold expansion improvements of the partonic cross sections [70 73] and of the pair-invariant mass distribution [74, an analysis of quasi-bound-state effects in $\sigma_{t \bar{t}}$ at the production threshold [75,76], determination of various building blocks for the computation of $\sigma_{t \bar{t}}$ at NNLO QCD [77,83], phenomenological analyses of boosted top-quark events [84,85] and of the use of the LHC $t \bar{t}$ cross section for calibrating parton distribution functions [86]. The NLO QCD Monte-Carlo generators [87, 88], [89], and [90] are important tools for the simulation of $t \bar{t}$ production and decay including parton showering [87,90]. Recently, results were presented based on a code which incorporates NLO QCD corrections to $t \bar{t}$ production and semileptonic top-quark decay with $t \bar{t}$ spin correlations [91].

In this paper we analyze a number of distributions and correlations, mostly for dileptonic $t \bar{t}$ partonic final states, at next-to-leading order in the strong and weak couplings. The spin-dependent terms are included to this order of the perturbation expansion in the production and decay matrix elements for the $t \bar{t}$ intermediate state. For our analysis we made a computer code with input based on previous work [13, 14, 19 21, 34 where the authors of this paper were involved. As our approach is based on spin density matrices being independent of specific spin reference axes, we can switch off the $t$ - and/or $\bar{t}$-spin dependent terms in the matrix elements, in particular the $t \bar{t}$

\footnotetext{
${ }^{1}$ For more complete overviews, cf., e.g., [37 39].
} 
spin correlations, also at NLO in the gauge couplings. This seems to us quite useful for performing comparative studies of final state distributions for correlated and uncorrelated $t \bar{t}$ events. This paper addresses the following issues: i) A study of the size of the weak interaction corrections with respect to NLO QCD corrections for several distributions and spin correlations. So far, only comparisons with respect to LO QCD results were made in the above-mentioned literature. ii) An investigation of the top-quark charge asymmetry and of two leptonic charge asymmetries [44] for correlated and uncorrelated dileptonic $t \bar{t}$ events at the Tevatron if acceptance cuts are applied. To our knowledge, this has not been analyzed so far to NLO in the gauge couplings. iii) A detailed analysis of dileptonic angular correlations induced by $t \bar{t}$ spin correlations.

In Sect. II we describe the ingredients of our analysis and list our input parameters. Sect. III contains results for several distributions and spin correlations at the level of the $t \bar{t}$ intermediate states. The top-quark charge asymmetry at the Tevatron will be considered in some detail and a comparison with the results of [45] will be made. In Sect. IV] we give our results for several distributions and angular correlations for dileptonic final states with phase-space cuts at NLO in the strong and weak couplings. Two distributions will be computed for semileptonic final states. We analyze the top-quark charge asymmetry and a related pair asymmetry at the Tevatron and two related leptonic charge asymmetries for correlated and uncorrelated dileptonic $t \bar{t}$ events, and we compare the top-quark asymmetries with experimental results [47 49]. Our predictions of the leptonic asymmetries have to await confrontation with measurements. Then we investigate a number of dileptonic angular correlations, for the Tevatron and the LHC at $\sqrt{s}=10$ and 14 $\mathrm{TeV}$, at NLO in the gauge couplings that are (in)sensitive to $t \bar{t}$ spin correlations. Moreover, we compare with the recent Tevatron measurements [65 67. Based on our SM results we identify, in addition, several observables that allow for searches for non-standard parity- and CP-violating interactions, especially with future LHC data. Sect. $\nabla$ contains a summary and outlook.

\section{Theoretical set-up}

We analyze hadronic top quark pair production and decay, at NLO in the QCD and weak couplings, in the on-shell approximation for the $t, \bar{t}$ quarks, taking their spin degrees of freedom in the production and decay stage into account. To be specific we consider, at the parton level, the following reactions:

$$
\begin{gathered}
g g, q \bar{q} \stackrel{t \bar{t}}{\longrightarrow} b \bar{b}+4 f, \\
g g, q \bar{q} \stackrel{t \bar{t}}{\longrightarrow} b \bar{b}+4 f+g, \\
g+q(\bar{q}) \stackrel{t \bar{t}}{\longrightarrow} b \bar{b}+4 f+q(\bar{q}),
\end{gathered}
$$

where $f=q, \ell, \nu_{\ell}$. As the top quark is a narrow resonance, we employ the narrow width approximation $\Gamma_{t} / m_{t} \rightarrow 0$ and incorporate the factorizable QCD and mixed weak-QCD corrections, which form gauge-invariant sets. In this approximation the squared matrix element $|\mathcal{M}|^{2}$ of the respective reaction is of the form

$$
|\mathcal{M}|^{2}=N_{i} \frac{1}{2 \hat{s}} \frac{\pi^{2}}{m_{t}^{2} \Gamma_{t}^{2}} \operatorname{Tr}[\rho R \bar{\rho}]=N_{i} \frac{1}{2 \hat{s}} \frac{\pi^{2}}{m_{t}^{2} \Gamma_{t}^{2}} \rho_{\alpha^{\prime} \alpha} R_{\alpha \alpha^{\prime}, \beta \beta^{\prime}} \bar{\rho}_{\beta^{\prime} \beta} .
$$


Here $R, \rho, \bar{\rho}$ are the production and $t$ and $\bar{t}$ decay density matrices for (II.1) - (II.3). The subscripts in Eq. (II.4) denote the top and antitop spin indices, and the factor $N_{i}$ arises from averaging over the colors and spins of the initial partons.

As already mentioned above, in our spin density matrix approach the $t, \bar{t}$ spin-dependent terms, in particular $t \bar{t}$ spin correlations can be switched off also at NLO in the gauge couplings. In addition, top-spin effects can be analyzed for arbitrary spin bases at NLO, in particular at the level of the $t \bar{t}$ intermediate states. This is quite useful for studies of non-standard interactions that change the SM-induced top-spin correlations.

As to the density matrices $R$ of the $t \bar{t}$ production reactions $q \bar{q} \rightarrow t \bar{t}(g), g g \rightarrow t \bar{t}(g), g q \rightarrow t \bar{t} q$, and $g \bar{q} \rightarrow t \bar{t} \bar{q}$ : Besides taking into account the $\mathcal{O}\left(\alpha_{s}^{3}\right)$ QCD corrections [14, we incorporate also the weak interaction corrections of order $\alpha_{s}^{2} \alpha, \alpha_{s} \alpha, \alpha^{2}$, and $\alpha_{s} \alpha^{2}$ to the spin density matrices of these processes as computed in [1921].

We use the decay density matrices of the main semileptonic and non-leptonic SM decay modes of polarized (anti)top quarks, $t \rightarrow b W^{+} \rightarrow b \ell^{+} \nu_{\ell}(g), b q \bar{q}^{\prime}(g)$ (where $\left.q \bar{q}^{\prime}=u \bar{d}, c \bar{s}\right)$ to $\mathcal{O}\left(\alpha_{s}\right)$ in fully differential form. Specific distributions were given in 34].

In the numerical implementation of (II.4) we use the expanded form of the right-hand side to NLO in the gauge couplings. We expand also the top width $\Gamma_{t}$ in the denominator of (II.4) to $\mathcal{O}\left(\alpha_{s}\right)$. In Sections III and IV below, the acronyms LO and NLO refer to the $\mathcal{O}\left(\alpha_{s}^{2}\right)$ and $\mathcal{O}\left(\alpha_{s}^{3}\right)$ matrix elements in QCD, while NLOW refers to the additional inclusion of the above-mentioned weak corrections.

The infrared (i.e. soft and collinear) singularities in the production density matrices are taken care of by a phase-space slicing method, as worked out explicitly in [14]. For semileptonic topquark decay we use a slicing procedure, too, while for non-leptonic decay a hybrid scheme is employed (cf. [34]). We checked that the results of Sections III and IV] are independent of the slicing parameter $x_{\text {min }}$ for $x_{\text {min }} \lesssim$ a few $\times 10^{-3}$. The results of these sections were obtained with $x_{\text {min }}=10^{-3}$.

With these building blocks we developed a numerical program for computing observables to fixed order in the gauge couplings as described above, at the level of the leptonic and partonic final states that correspond to the dileptonic, lepton plus jets, and all-jets final states. As we are primarily interested in modeling "top as a signal", we investigate in this paper only distributions mostly for dileptonic and a few for semileptonic final states which have the highest signal-to-background ratios.

$$
\begin{aligned}
& p \bar{p}, p p \quad \rightarrow t \bar{t}+X \rightarrow \ell^{+} \ell^{\prime-} j_{b} j_{\bar{b}}+X, \\
& p \bar{p}, p p \quad \rightarrow t \bar{t}+X \rightarrow \ell^{+} j_{b} j_{\bar{b}} j_{1} j_{2}+X,
\end{aligned}
$$

where $\ell=e, \mu, \tau$, and $j_{b}\left(j_{1,2}\right)$ denote (non) $b$ jets.

Our input parameters are as follows. Throughout this paper the top-quark mass $m_{t}$ is defined in the on-shell scheme. Adopting the common, albeit not rigorously justifiable practice of identifying the experimentally determined top mass with the on-shell mass, we use for $m_{t}$ the recent Tevatron average $m_{t}=173.1 \pm 1.3 \mathrm{GeV}$ [92]. Our main results, the charge asymmetries and the top-spin induced angular distributions and correlations given in Sect. IV are, in fact, not very sensitive to variations $\Delta m_{t}$ of the top mass by a few GeV. The QCD coupling is defined, as usual, in the $\overline{\mathrm{MS}}$ scheme. We employ CTEQ6L1 and CTEQ6.6M parton distribution functions (PDF) for LO and 
NLO computations [86], which correspond to $\alpha_{s}\left(m_{Z}\right)=0.130$ and $\alpha_{s}\left(m_{Z}\right)=0.118$, respectively. The evolution of $\alpha_{s}=\alpha_{s, 5}$ to some higher scale $\mu_{R}$ is made within 5-flavor QCD. In the parton matrix elements, which were computed in [14] within 6-flavor QCD, the $\overline{\mathrm{MS}}$ coupling $\alpha_{s, 6}\left(\mu_{R}\right)$ is replaced by $\alpha_{s, 5}\left(\mu_{R}\right)$ according to $\alpha_{s, 6} / \pi=\alpha_{s, 5} / \pi-\frac{1}{6}\left(\alpha_{s, 5} / \pi\right)^{2} \ln \left(m_{t}^{2} / \mu_{R}^{2}\right)+\mathcal{O}\left(\alpha_{s, 5}^{3}\right)$ [93]. For the top width we use $\Gamma_{t}=1.3882-\alpha_{s}\left(m_{t}\right) \times 0.8076 \mathrm{GeV}$. We use an on-shell mass $m_{b}=4.8 \mathrm{GeV}$ for the $b$ quark, while the masses of the lighter quarks and of the leptons are put equal to zero. In the evaluation of the weak corrections we use the QED coupling $\alpha\left(m_{Z}\right)=0.008$ and for the $W$ and $Z$-boson masses and the $W$-boson width $\Gamma_{W}$ the measured values given in [94. We put the CKM matrix elements that appear in the CKM-unsuppressed semi- and non-leptonic top-quark decay amplitudes equal to one, $\left|V_{t b}\right|=\left|V_{q q^{\prime}}\right|=1$. The Higgs-boson mass is put equal to $m_{H}=120$ $\mathrm{GeV}$. The dependence of the results of Sections III and IV on $m_{H} \lesssim 200 \mathrm{GeV}$ is insignificant.

\section{Results for $t \bar{t} X$ :}

In this section we give results, at LO, NLO, and NLOW in the gauge couplings, for several distributions and correlations at the level of on-shell $t \bar{t}$ intermediate states,

$$
p \bar{p}, p p \rightarrow t \bar{t}+X
$$

both for the Tevatron $(\sqrt{s}=1.96 \mathrm{TeV})$ and for the LHC at center-of-mass energies $\sqrt{s}=10$ and $14 \mathrm{TeV}$. No phase space cuts are applied in this section. The factorization and renormalization scales are put equal, $\mu_{F}=\mu_{R} \equiv \mu$ and we choose $\mu=m_{t} / 2, m_{t}$, and $2 m_{t}$.

As was shown in [20], the weak-interaction contributions to the total cross section $\sigma_{t \bar{t}}$ at the Tevatron and at the LHC are marginal as compared with the NLO QCD corrections. On the other hand, for a number of distributions, for instance, the transverse momentum distribution of the (anti)top quark and the $t \bar{t}$ invariant mass distribution, the weak-interaction corrections are of potential importance at large energies due to large Sudakov logarithms. For the $p_{T}\left(M_{t \bar{t}}\right)$ distribution at the LHC these corrections amount to about $-10 \%(-6 \%)$ of the LO results for $p_{T} \sim 1 \mathrm{TeV}\left(M_{t \bar{t}} \sim 2 \mathrm{TeV}\right)$, and the respective ratios $d \sigma_{\text {weak }} / d \sigma_{L O}$ grow in magnitude for larger $p_{T}$ and $M_{t \bar{t}}$ [19 23]. Here we compare the weak and NLO QCD corrections for $t \bar{t}$ production, to wit, for the $p_{T}$ and $M_{t \bar{t}}$ distributions and for several $t \bar{t}$ spin correlation observables at the LHC, and for the charge asymmetry at the Tevatron.

Fig. 1 shows, for the LHC at $\sqrt{s}=14 \mathrm{TeV}$, the (un)normalized $t \bar{t}$ invariant-mass distribution at LO, NLO, and NLOW for $\mu=m_{t}$. The analogous plots are displayed in Fig. 2 for the (un)normalized $p_{T}$ distribution of the top quark. In Fig. 3 the ratios of these distributions evaluated at NLOW and NLO are plotted for $\mu=m_{t}$. The weak-interaction corrections to the $M_{t \bar{t}}$ distribution are negative except close to $2 m_{t}$ and become larger in magnitude than $\sim 2 \%$ with respect to the NLO QCD corrections for $M_{t \bar{t}} \gtrsim 1.2 \mathrm{TeV}$. For the $p_{T}$ distribution the respective ratio is also smaller than one, except in the extreme forward- and backward region [21], and the weak-interaction corrections grow to several percent in magnitude beyond $p_{T} \sim 500 \mathrm{GeV}$ (not shown in Fig. 3). A study of the scale dependence of the normalized $p_{T}$ and $M_{t \bar{t}}$ distributions in the range $p_{T} \lesssim 1 \mathrm{TeV}, M_{t \bar{t}} \lesssim 1.2 \mathrm{TeV}$ shows that for $m_{t} / 2 \leq \mu \leq 2 m_{t}$ the scale variations are of the same order of magnitude as the weak-interaction contributions to the NLO QCD distributions. 

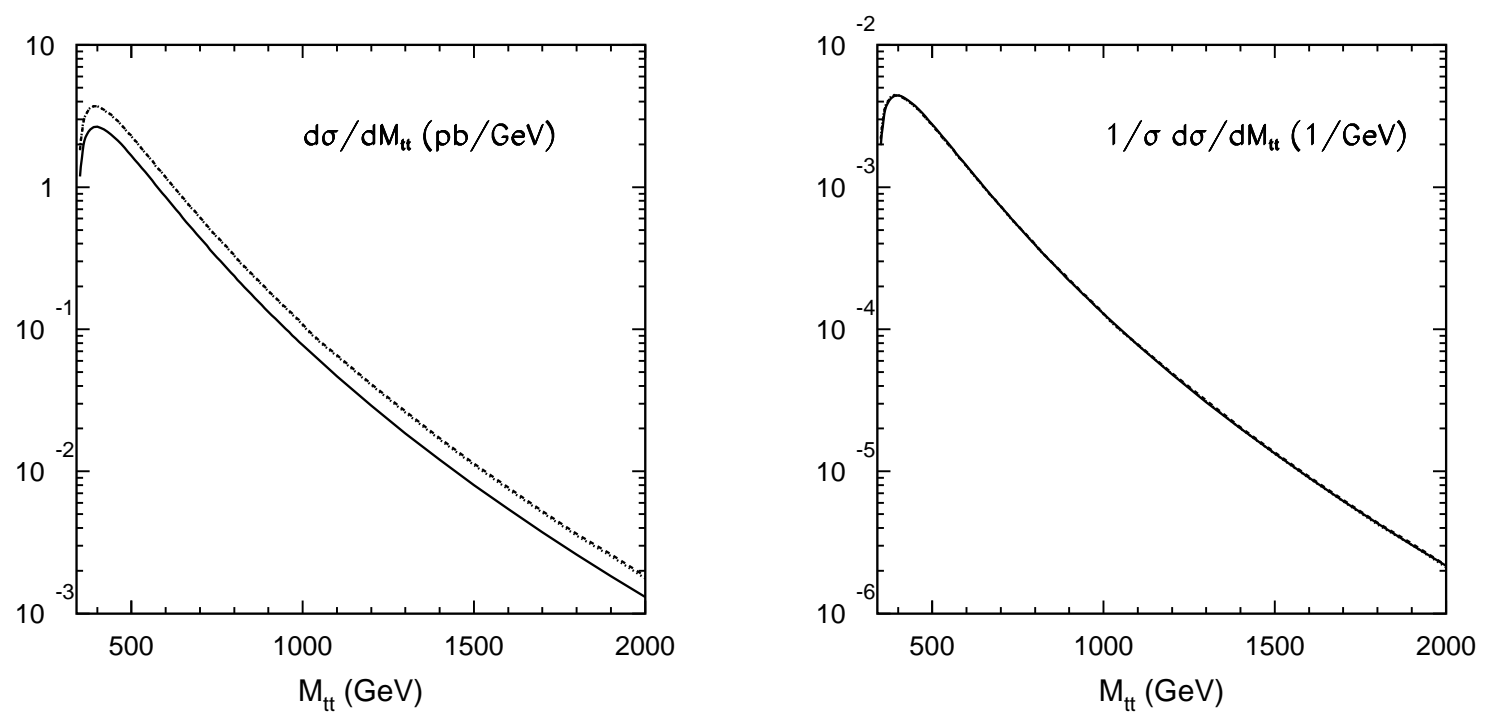

Figure 1: The unnormalized (left panel) and normalized (right panel) $t \bar{t}$ invariant-mass distribution for the LHC $(\sqrt{s}=14 \mathrm{TeV})$ at LO (solid), NLO (dashed), and NLOW (dotted) for $\mu=m_{t}$.
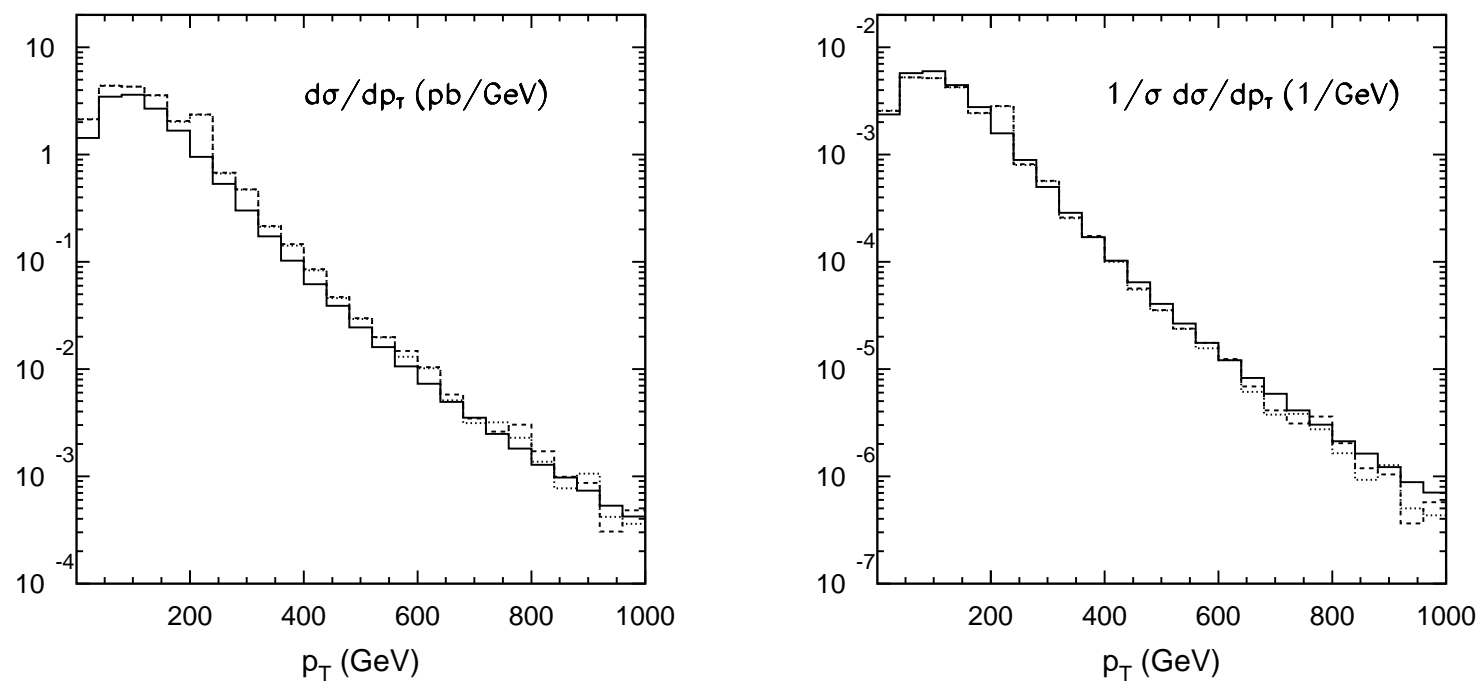

Figure 2: The unnormalized (left) and normalized (right) $p_{T}$ distribution of the top quark for the LHC $(\sqrt{s}=14 \mathrm{TeV})$ at LO (solid), NLO (dashed), and NLOW (dotted) for $\mu=m_{t}$. 

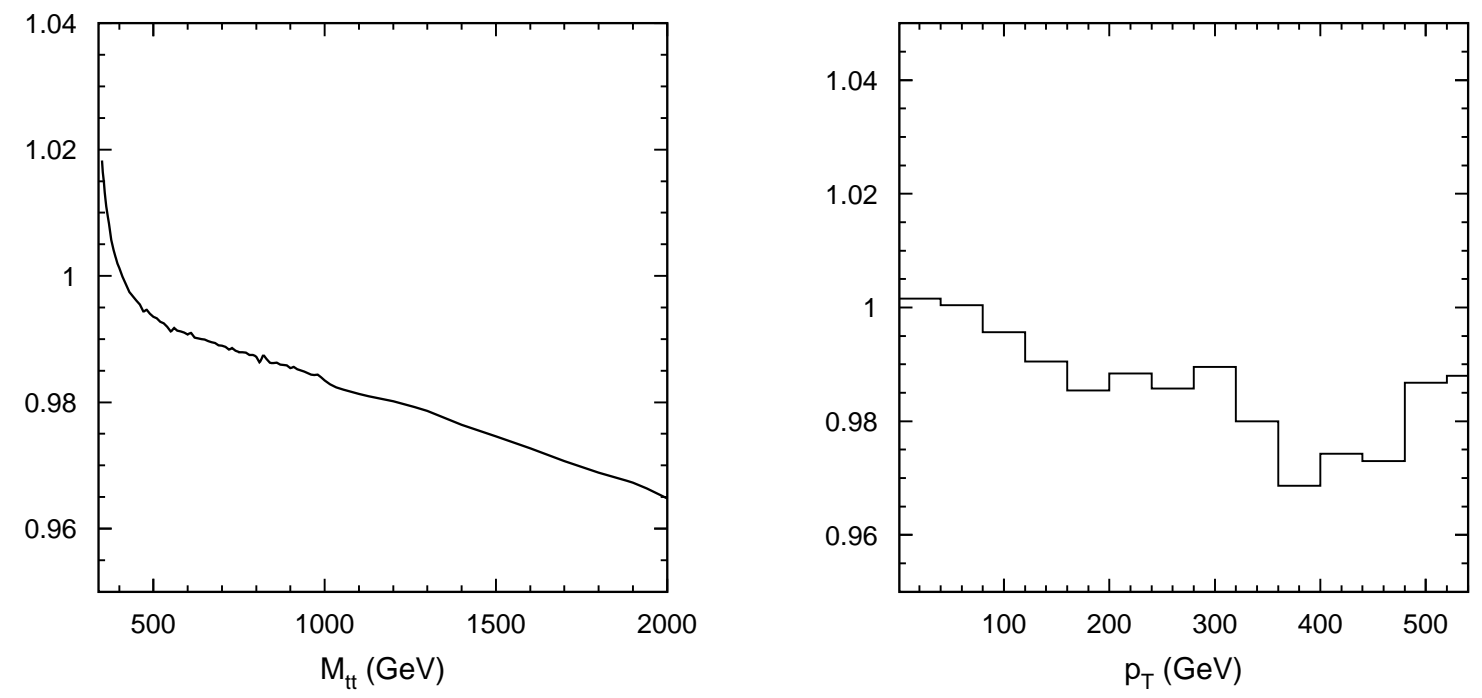

Figure 3: The ratio of the $M_{t \bar{t}}$ distribution evaluated at NLOW and NLO with $\mu=m_{t}$ (left panel) and the corresponding ratio of the $p_{T}$ distribution (right panel) for the LHC $(14 \mathrm{TeV})$.

Next we consider the asymmetry with respect to interchange of the the $t$ and $\bar{t}$ charges, which is known to be relatively large at the Tevatron. This asymmetry is generated at NLO QCD by the interference of even and odd terms under $t \leftrightarrow \bar{t}$ in the amplitudes for $q \bar{q}$ annihilation and, likewise, for $g q$ and $g \bar{q}$ fusion [2,4,42 46]. (The contribution of the latter two processes to the charge asymmetry is one order of magnitude below the $q \bar{q}$ contribution.) The weak interaction corrections to the $q \bar{q}$ and flavor excitation processes contribute, too. The conventionally defined differential and integrated charge asymmetry, $A(y)$ and $A$, are:

$$
A(y)=\frac{N_{t}(y)-N_{\bar{t}}(y)}{N_{t}(y)+N_{\bar{t}}(y)}, \quad A=\frac{\int_{y>0} N_{t}(y)-\int_{y>0} N_{\bar{t}}(y)}{\int_{y>0} N_{t}(y)+\int_{y>0} N_{\bar{t}}(y)},
$$

where $y$ denotes the rapidities $y_{t}, y_{\bar{t}}$ of the $t$ and $\bar{t}$ quark defined in the laboratory frame, and $N(y)=d \sigma_{t \bar{t}} / d y$.

As the $p \bar{p}$ state at the Tevatron is a $\mathrm{CP}$ eigenstate in the laboratory frame, $\mathrm{CP}$ invariance of the SM corrections 2 implies that $N_{\bar{t}}\left(y_{\bar{t}}\right)=N_{t}\left(-y_{t}\right)$, which in turn implies that $A$ is equal to the forward-backward asymmetry of the top quark:

$$
A_{F B}^{t}=\frac{\int_{y>0} N_{t}(y)-\int_{y<0} N_{t}(y)}{\int_{y>0} N_{t}(y)+\int_{y<0} N_{t}(y)} .
$$

\footnotetext{
${ }^{2}$ The CP-violating terms in the matrix elements for $p \bar{p} \rightarrow t \bar{t} \rightarrow$ final state induced by the non-zero SM KM phase $\delta_{K M}$ in higher orders of perturbation theory are of course numerically irrelevant.
} 
In the case of the Tevatron, $C P$ invariance tells us also that $A_{F B}^{\bar{t}}=-A_{F B}^{t}$. Non-standard $C P$ violating interactions, if existent, may invalidate this relation by a small amount. As to $t \bar{t}$ produced by $g g$ fusion: the Bose symmetry of the $g g$ state precludes a contribution from this sub-process to the integrated asymmetries $A, A_{F B}^{t}$ - irrespective of whether or not the production density matrix $R_{g g}$ contains $\mathrm{P}$ - and/or CP-violating pieces.

The D0 and CDF experiments [47,48] and [45] considered the following observable which also reflects the charge asymmetry in $t \bar{t}$ production at the Tevatron:

$$
A^{t \bar{t}}=\frac{\int N(\Delta y>0)-\int N(\Delta y<0)}{\int N(\Delta y>0)+\int N(\Delta y<0)},
$$

where $\Delta y=y_{t}-y_{\bar{t}}$. This asymmetry is, for kinematical reasons, larger than $A$.

It should be recalled that the numerators of the above asymmetries start at order $\alpha_{s}^{3}$ in the QCD perturbation expansion. Thus, the above inclusive asymmetries are of order $\alpha_{s}$. They were computed to this order, including weak corrections, by [43, 45]. The order $\alpha_{s}^{4}$ QCD corrections are not known. Also within our framework, the determination of these asymmetries is a LO calculation. However, we compute here the numerators of $A$ and $A^{t \bar{t}}$ by taking into account the NLO respectively NLOW $t \bar{t}$ parton matrix elements 3 both from $q \bar{q}, g q(\bar{q})$, and $g g$ initial states. (In this way, the computation of these asymmetries provides also a check of our code.) We adopt the following procedure. We evaluate the numerators at NLO and NLOW, respectively, with NLO PDF and the denominators at LO with LO PDF, using however the same value of $\alpha_{s}$ in both numerator and denominator. (Evaluating the denominator including the NLO corrections would be in conflict with the perturabtion expansion.) We label our results with the acronyms NLO' and NLOW', respectively.

We have plotted in Fig. 4 the differential charge asymmetry (III.2) for the Tevatron at NLO' and NLOW' for a fixed scale, $\mu=m_{t}$. Table 1 contains our results for the integrated charge asymmetry $A$ and the pair asymmetry $A^{t \bar{t}}$ for the for three different scales.

Table 1: Results for the charge asymmetry $A$ and the pair asymmetry $A^{t \bar{t}}$ at NLO' and NLOW' for $\mu=m_{t} / 2, m_{t}$, and $2 m_{t}$. The acronyms NLO' and NLOW' are explained in the text.

\begin{tabular}{||c|c|c|c||}
\hline \hline & \multicolumn{3}{|c|}{ Tevatron } \\
\hline \hline$\mu$ & $m_{t} / 2$ & $m_{t}$ & $2 m_{t}$ \\
\hline \hline$A$ (NLO') & 0.054 & 0.049 & 0.045 \\
\hline$A$ (NLOW') $^{\prime \prime}$ & 0.056 & 0.051 & 0.048 \\
\hline \hline$A^{t t}$ (NLO') & 0.084 & 0.076 & 0.071 \\
\hline$A^{t t}$ (NLOW') & 0.087 & 0.080 & 0.075 \\
\hline \hline
\end{tabular}

\footnotetext{
${ }^{3}$ In the calculation of [43, 45] just the relevant $\mathcal{O}\left(\alpha_{s}^{3}\right)$ terms that generate the asymmetries were taken into account in the numerator. That is why they used LO PDF also in the numerator.
} 


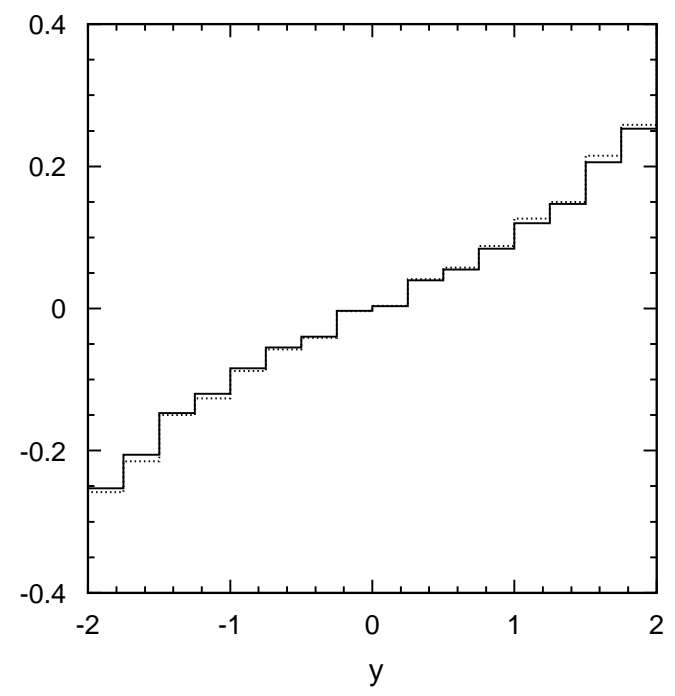

Figure 4: The differential charge asymmetry $A(y)$, eq. (피.2), for the Tevatron as a function of the (anti)top-quark rapidity $y$, for $\mu=m_{t}$. The solid and dotted lines correspond to NLO' and NLOW', respectively.

Our results for $A$ and $A^{t \bar{t}}$ given in Table 1 agree with the results $A=0.051(6)$ and $A^{t \bar{t}}=0.078(9)$ of [45]. The following remarks are, however, in order. We use a different set of PDF and a slightly different value of $m_{t}$. The contribution of the mixed electroweak-QCD corrections to the charge asymmetries were determined in [43] for the $q \bar{q}$ initiated production process which, as found by these authors, increases the QCD asymmetry $A$ by a factor 1.09. We have taken into account in addition the weak-interaction contributions to $g q(\bar{q}) \rightarrow t \bar{t} q(\bar{q})$ and a specific contribution to $b \bar{b} \rightarrow t \bar{t}[21]$. In Born approximation, the amplitude of the latter reaction consists of $t$-channel $W$ boson exchange $b \bar{b} \stackrel{W}{\longrightarrow} t \bar{t}$ and $s$-channel photon, $Z$ boson, and gluon exchanges $b \bar{b} \stackrel{\gamma, Z, g}{\longrightarrow} t \bar{t}$. The terms in $|\mathcal{M}(b \bar{b} \rightarrow t \bar{t})|_{B}^{2}$ that involve the electroweak interactions are of order $\alpha^{2}$ and $\alpha_{s} \alpha$, respectively. They contain also charge-asymmetric pieces which contribute to $A$ and $A^{t \bar{t}}$ with opposite sign as compared to the contributions from the order $\alpha_{s}^{2} \alpha$ terms in the $q \bar{q} \rightarrow t \bar{t}$ matrix element. At present the size of these $b \bar{b}$ induced contributions can not be determined precisely in view of the rather poorly known $b$-quark PDF. In any case, these additional terms diminish the overall size of the weak-interaction contributions, as the numbers of Table 1 show.

The spread of the asymmetries in Table1 1ue to scale variations certainly underestimates the true uncertainties of these predictions of the inclusive asymmetries 4 . A more realistic estimate of the theory uncertainty, namely $\sim 30 \%$, is provided by the calculation of $A$ in [46] where NLL QCD threshold resummation corrections were included. Comparisons with the experimental results from the Tevatron will be made in the next section.

\footnotetext{
${ }^{4}$ For $t \bar{t}+$ jet final states the charge asymmetry was calculated to NLO QCD in 27 .
} 
At the LHC, the initial $p p$ state is an eigenstate of parity. Thus, $A_{F B}^{t}=A_{F B}^{\bar{t}}=0$ in the laboratory frame, as long as only parity-invariant interactions - more general, only parity-even terms in the scattering operator - are taken into account. The parity-violating terms of the weak corrections appear in fact only in the $t$ - and/or $\bar{t}$-spin dependent terms of the partonic production density matrices and do, therefore, not contribute when making predictions for top quarks summed over their spins. As a consequence, the differential charge asymmetry $A(y)$ at the LHC induced by the SM interactions must be symmetric with respect to $y=0$. Thus, in the absence of cuts, the integrated charge asymmetry $A=0$. We have checked that our code reproduces this conclusion. Nevertheless, there are non-zero effects in suitably defined distributions [43, 45].

Next we consider top-spin induced effects. The polarization of the $t$ or $\bar{t}$ quarks in the hadronically produced $t \bar{t}$ sample due to weak interactions or QCD absorptive parts is small [20,58]. On the other hand, the QCD dynamics leads to correlations of the $t, \bar{t}$ spins already at tree level which are characteristic of the production mode. One may investigate $t \bar{t}$ spin correlations by the following expectation values of spin operators $\mathbf{O}$ :

$$
\mathcal{O}_{a b}=\left\langle 4\left(\mathbf{S}_{t} \cdot \hat{\mathbf{a}}\right)\left(\mathbf{S}_{\bar{t}} \cdot \hat{\mathbf{b}}\right)\right\rangle \quad \text { and } \quad \mathcal{O}_{\text {spin }}=\left\langle\frac{4}{3} \mathbf{S}_{t} \cdot \mathbf{S}_{\bar{t}}\right\rangle
$$

Here $\mathbf{S}_{t}, \mathbf{S}_{\bar{t}}$ are the top and antitop spin operators, and the unit vectors $\hat{\mathbf{a}}$ and $\hat{\mathbf{b}}$ are arbitrary reference directions, which may be interpreted as spin axes. As usual, the normalization of the averages is such that $\langle 1\rangle=1$. We use the following spin bases [14] in the zero-momentum frame $(\mathrm{ZMF})$ of the $t \bar{t}$ pair:

$$
\begin{array}{rlrl}
\hat{\mathbf{a}}=-\hat{\mathbf{b}} & =\hat{\mathbf{k}}, & & \text { (helicity basis) } \\
\hat{\mathbf{a}}=\hat{\mathbf{b}}=\hat{\mathbf{p}}, & & \text { (beam basis) } \\
\hat{\mathbf{a}}=\hat{\mathbf{b}}=\hat{\mathbf{d}}, & & \text { (off-diagonal basis). }
\end{array}
$$

Here $\hat{\mathbf{k}}$ denotes the direction of flight of the top quark in the $t \bar{t}$-ZMF and $\hat{\mathbf{p}}$ is the direction of one of the colliding hadrons in that frame. The unit vector $\hat{\mathbf{d}}$, which we use in the convention of [14, defines the so-called off-diagonal basis [60]. The correlations $\mathcal{O}_{a b}\left(\mathcal{O}_{\text {spin }}\right)$ are equivalent to (a sum of) double spin asymmetries. At the Tevatron, the strength of the spin correlations induced by the SM interactions is largest with respect to the beam and off-diagonal basis. For the LHC the helicity correlation and $\mathcal{O}_{\text {spin }}$ are good choices 5 .

We consider the variation of these correlations with $M_{t \bar{t}}$, which we define by

$$
\frac{d \mathcal{O}}{d M_{t \bar{t}}}=\frac{1}{\sigma} \int d \sigma \mathbf{O} \delta\left(\sqrt{\left(p_{t}+p_{\bar{t}}\right)^{2}}-M_{t \bar{t}}\right) .
$$

We normalize to the integrated cross section.

The distributions are displayed in Fig. 5 to LO, NLO, and NLOW for the beam and off-diagonal basis at the Tevatron. For the helicity correlation and for $\mathcal{O}_{\text {spin }}$ the analogous plots are given in Fig. 6 for the LHC $(14 \mathrm{TeV})$. The latter distributions pass through zerd 6 near $M_{t \bar{t}} \sim 800 \mathrm{GeV}$.

\footnotetext{
${ }^{5}$ For $g g \rightarrow t \bar{t}$ an optimal axis was constructed in [61. It was reconsidered recently in [64]. Cf. the next section.

${ }^{6}$ Their detailed behaviour can be read off from plots of scaling functions given in [14].
} 
These figures show the known feature that the $t \bar{t}$ spin correlations (III.5) at the Tevatron and LHC receive most of their contributions from $t \bar{t}$ pairs with rather low $M_{t \bar{t}}$. In Figs. 7 and 8 the respective distributions are given for $\mu=m_{t} / 2, m_{t}$, and $2 m_{t}$.

In order to quantify the size of the weak corrections we have plotted in Fig. 9 the ratios of $d \mathcal{O} / d M_{t \bar{t}}$ evaluated at NLOW and NLO for $\mathcal{O}_{\text {spin }}$ and $\mathcal{O}_{\text {hel }}$ at the LHC $(14 \mathrm{TeV})$. This figure shows that the weak-interaction corrections to $\mathcal{O}_{\text {spin }}$ are negative and $\lesssim 4 \%$ with respect to NLO QCD for $M_{t \bar{t}}<800 \mathrm{GeV}$, while these corrections are smaller for $\mathcal{O}_{\text {hel }}$. In the latter case, the increase of the magnitude of the ratio in the vicinity of $M_{t \bar{t}} \sim 800 \mathrm{GeV}$ is just a consequence of the NLO correlation passing through zero and changing sign afterwards.

In Table 2 the values of these variables, integrated over the $M_{t \bar{t}}$ spectrum, are given at NLOW for three scales. The spin correlations will be analyzed further for dileptonic final states in the next section. There we will also compare the numbers of Table 2 with the results of [14].

Table 2: Results for several spin correlation variables at NLOW at the level of $t \bar{t}$ final states (no cuts) for the Tevatron and LHC. At the LHC the SM-induced $t \bar{t}$ spin correlations are very small with respect to the beam and off-diagonal bases and are therefore not given. Their SM values were given in [13, 14].

\begin{tabular}{||c|c|c|c|c|c|c||c|c|c||}
\hline \hline & \multicolumn{3}{|c|}{ Tevatron } & \multicolumn{3}{c|}{ LHC $(10 \mathrm{TeV})$} & \multicolumn{3}{c||}{ LHC $(14 \mathrm{TeV})$} \\
\hline \hline$\mu$ & $m_{t} / 2$ & $m_{t}$ & $2 m_{t}$ & $m_{t} / 2$ & $m_{t}$ & $2 m_{t}$ & $m_{t} / 2$ & $m$ & $2 m$ \\
\hline \hline $\mathcal{O}_{\text {beam }}$ & 0.777 & 0.791 & 0.804 & & & & & & \\
\hline $\mathcal{O}_{\text {off }}$ & 0.783 & 0.798 & 0.810 & & & & & & \\
\hline \hline $\mathcal{O}_{\text {spin }}$ & 0.216 & 0.218 & 0.220 & -0.236 & -0.233 & -0.229 & -0.239 & -0.236 & -0.234 \\
\hline $\mathcal{O}_{\text {hel }}$ & -0.358 & -0.368 & -0.376 & 0.326 & 0.326 & 0.325 & 0.327 & 0.328 & 0.331 \\
\hline \hline
\end{tabular}



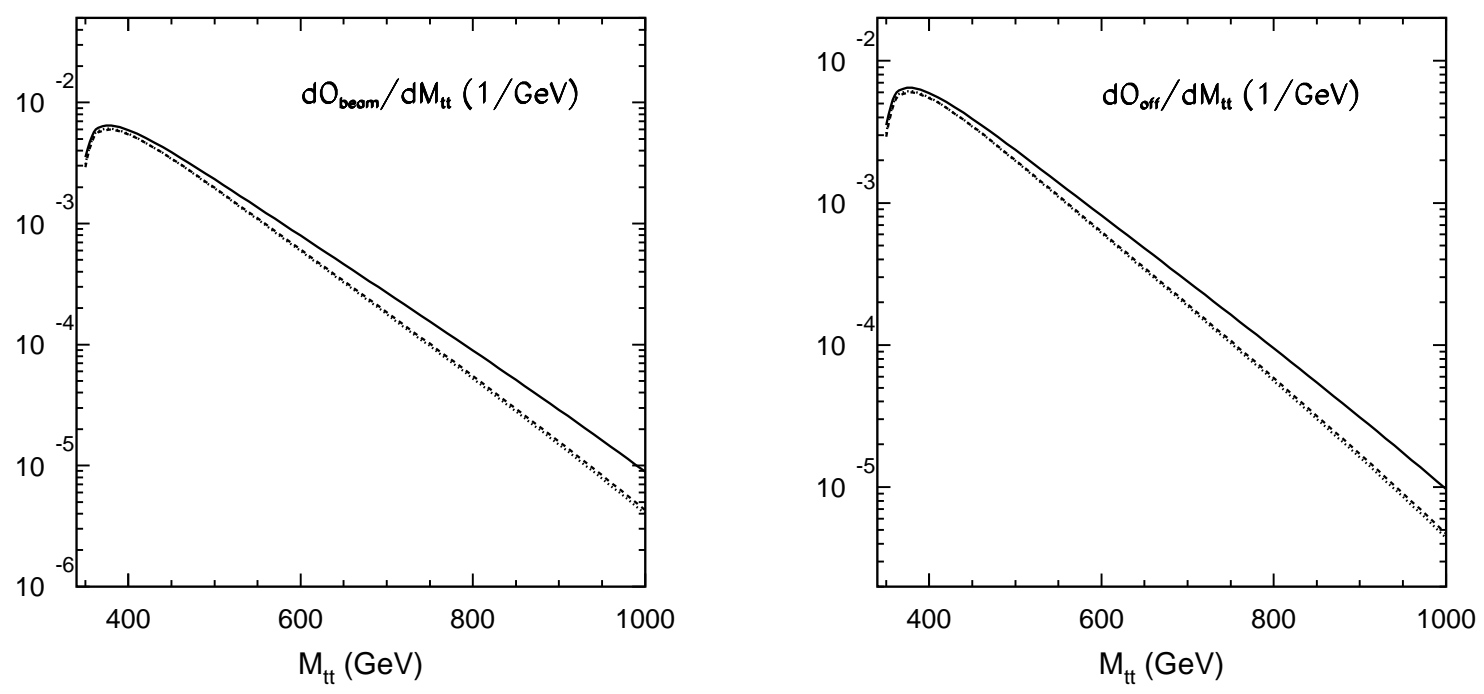

Figure 5: The distributions $d \mathcal{O}_{\text {beam }} / d M_{t \bar{t}}$ (left) and $d \mathcal{O}_{\text {off }} / d M_{t \bar{t}}$ (right) for the Tevatron at LO (solid), NLO (dashed), and NLOW (dotted) for $\mu=m_{t}$.
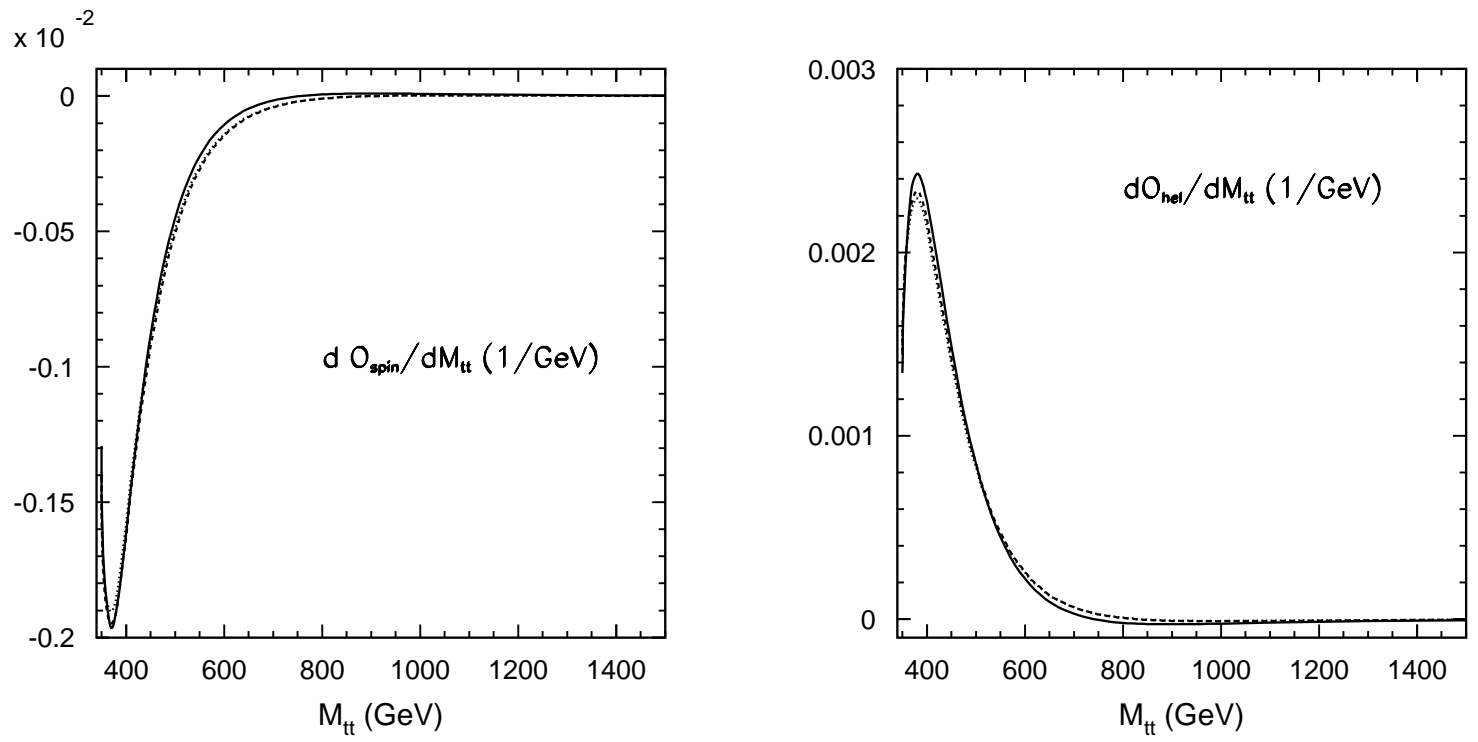

Figure 6: The distributions $d \mathcal{O}_{\text {spin }} / d M_{t \bar{t}}$ (left) and $d \mathcal{O}_{\text {hel }} / d M_{t \bar{t}}$ (right) for the $\operatorname{LHC}(\sqrt{s}=14 \mathrm{TeV})$ at LO (solid), NLO (dashed), and NLOW (dotted) for $\mu=m_{t}$. 

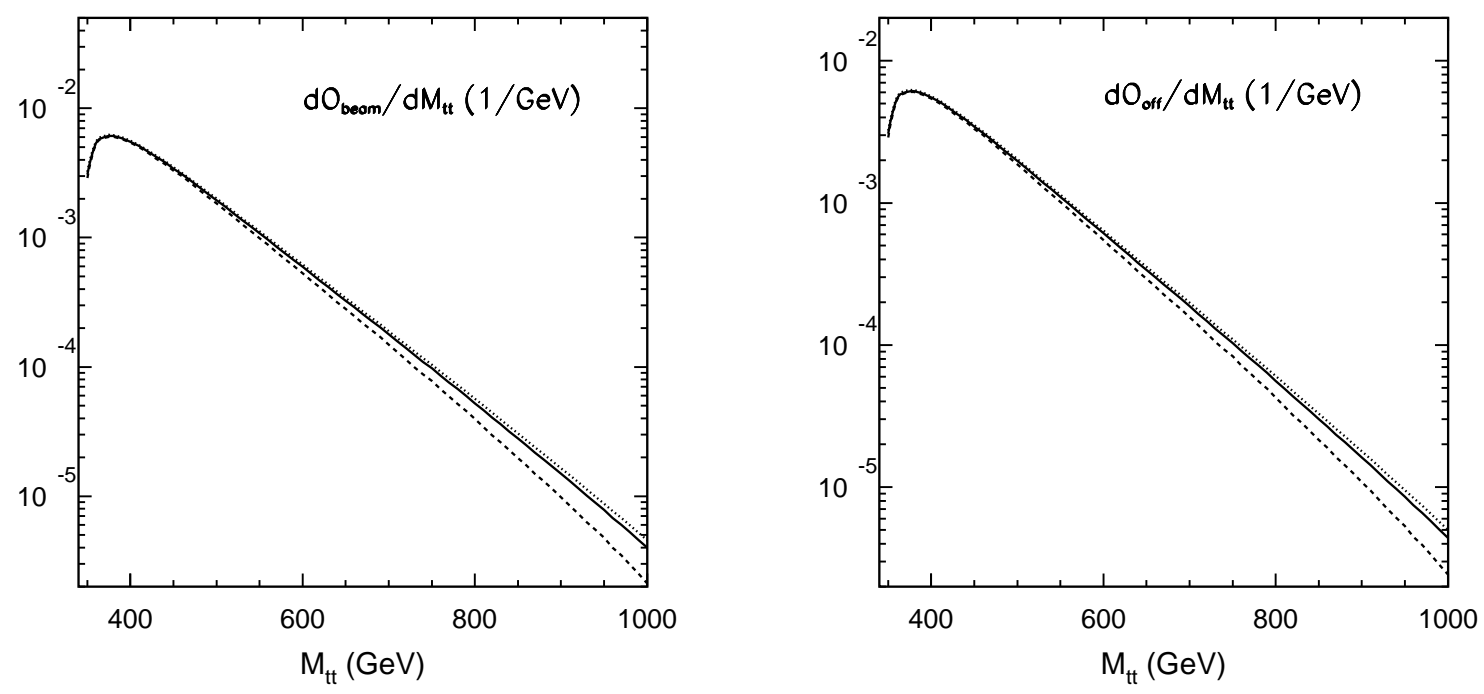

Figure 7: The distributions $d \mathcal{O}_{\text {beam }} / d M_{t \bar{t}}$ (left) and $d \mathcal{O}_{\text {off }} / d M_{t \bar{t}}$ (right) for the Tevatron at NLOW for $\mu=m_{t} / 2$ (dashed), $\mu=m_{t}$ (solid), and $\mu=2 m_{t}$ (dotted).
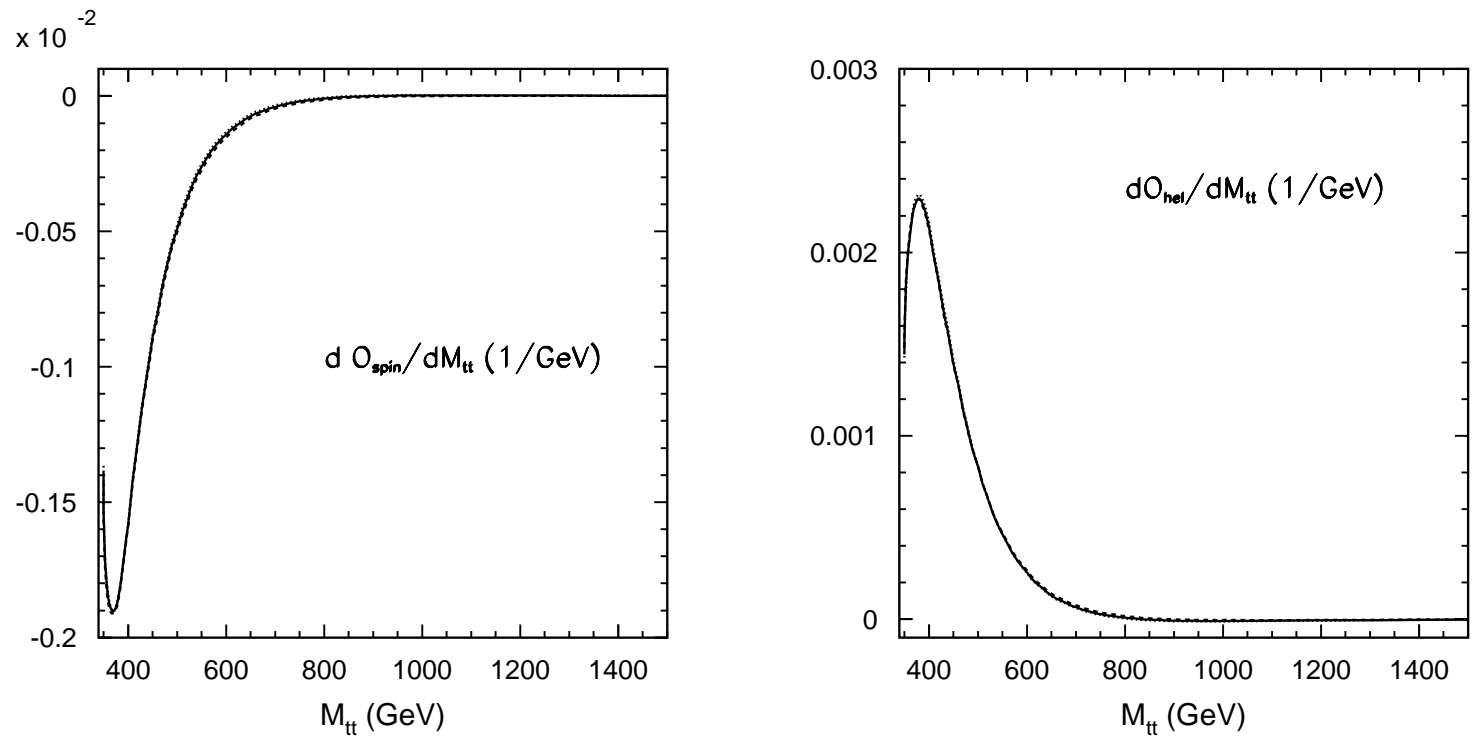

Figure 8: The distributions $d \mathcal{O}_{\text {spin }} / d M_{t \bar{t}}$ (left) and $d \mathcal{O}_{\text {hel }} / d M_{t \bar{t}}$ (right) for the $\mathrm{LHC}(\sqrt{s}=14 \mathrm{TeV})$ at NLOW for $\mu=m_{t} / 2$ (dashed), $\mu=m_{t}$ (solid), and $\mu=2 m_{t}$ (dotted). 

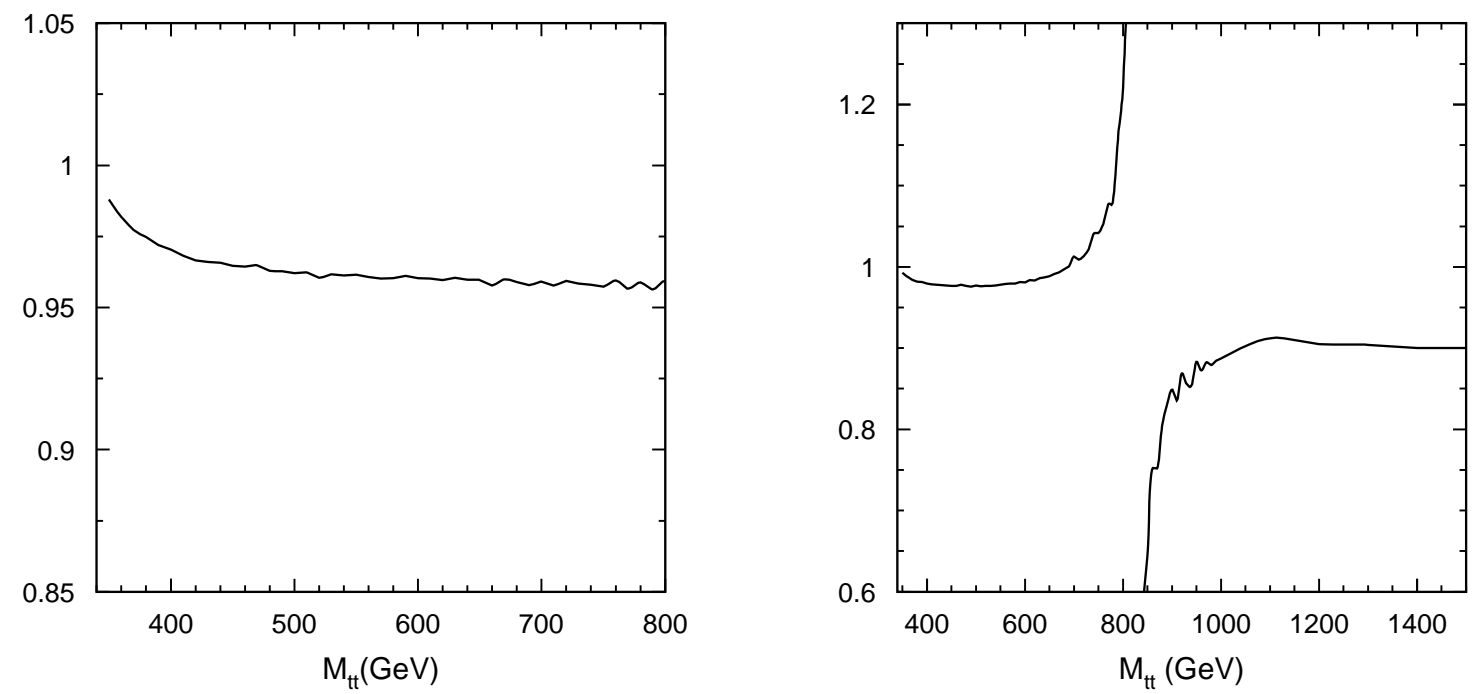

Figure 9: The ratios of $d \mathcal{O} / d M_{t \bar{t}}$ evaluated at NLOW and NLO with $\mu=m_{t}$ for $\mathcal{O}_{\text {spin }}$ (left panel) and $\mathcal{O}_{\text {hel }}$ (right panel) for the LHC $(14 \mathrm{TeV})$.

\section{Results for dilepton and semileptonic final states}

In this section we give results for several distributions, correlations, and asymmetries for dileptonic final states (II.6) , and for a few observables for semileptonic final states (II.5), at NLO in the strong and weak couplings (NLOW). The radiative corrections were implemented in our computer code as described in Section III.

We use the following cuts for the dileptonic final states $\left(\ell=e, \mu, E_{T}\right.$ denotes the missing transverse energy, and $\eta$ is the pseudorapidity):

Tevatron : $\quad p_{T}^{\ell} \geq 20 \mathrm{GeV}, \quad\left|\eta_{\ell}\right| \leq 2.0, \quad p_{T}^{j} \geq 20 \mathrm{GeV}, \quad\left|\eta_{j}\right| \leq 2.0, \quad E_{T} \geq 25 \mathrm{GeV}$,

$$
\mathrm{LHC}: \quad p_{T}^{\ell} \geq 20 \mathrm{GeV}, \quad\left|\eta_{\ell}\right| \leq 2.5, \quad p_{T}^{j} \geq 20 \mathrm{GeV}, \quad\left|\eta_{j}\right| \leq 2.4, \quad E_{T} \geq 40 \mathrm{GeV} .
$$

For the semileptonic $t \bar{t}$ final states we use

$\begin{aligned} \text { Tevatron : } & p_{T}^{\ell} \geq 20 \mathrm{GeV}, \quad\left|\eta_{\ell}\right| \leq 2.0, \quad p_{T}^{j} \geq 20 \mathrm{GeV}, \quad\left|\eta_{j}\right| \leq 2.0, \quad E_{T} \geq 20 \mathrm{GeV}, \\ \mathrm{LHC}: & p_{T}^{\ell} \geq 20 \mathrm{GeV}, \quad\left|\eta_{\ell}\right| \leq 2.5, \quad p_{T}^{j} \geq 20 \mathrm{GeV}, \quad\left|\eta_{j}\right| \leq 2.4, \quad \mathbb{E}_{T} \geq 20 \mathrm{GeV} .\end{aligned}$

We put again $\mu_{R}=\mu_{F}=\mu$ and evaluate the distributions for $\mu=m_{t} / 2, m_{t}$, and $2 m_{t}$. We calculated the observables below inclusively. We checked for some dilepton final-state distributions that the results do not change when using instead the $k_{\perp}$ jet algorithm [95]. This is to say that we checked an inclusive calculation against $\ell^{+} \nu_{\ell} \ell^{-} \bar{\nu}_{\ell} j_{b} j_{\bar{b}}$ (NLO) and $\ell^{+} \nu_{\ell} \ell^{-} \bar{\nu}_{\ell} j_{b} j_{\bar{b}} j$ (LO), where $j$ denotes a gluon or light quark jet.

Fig. 10 shows, for the dilepton channels at the Tevatron and LHC (10 TeV), the $p_{T}^{\ell}$ distribution of the charged lepton $\ell^{+}$for three scales $\mu$. We have normalized to the respective cross section 

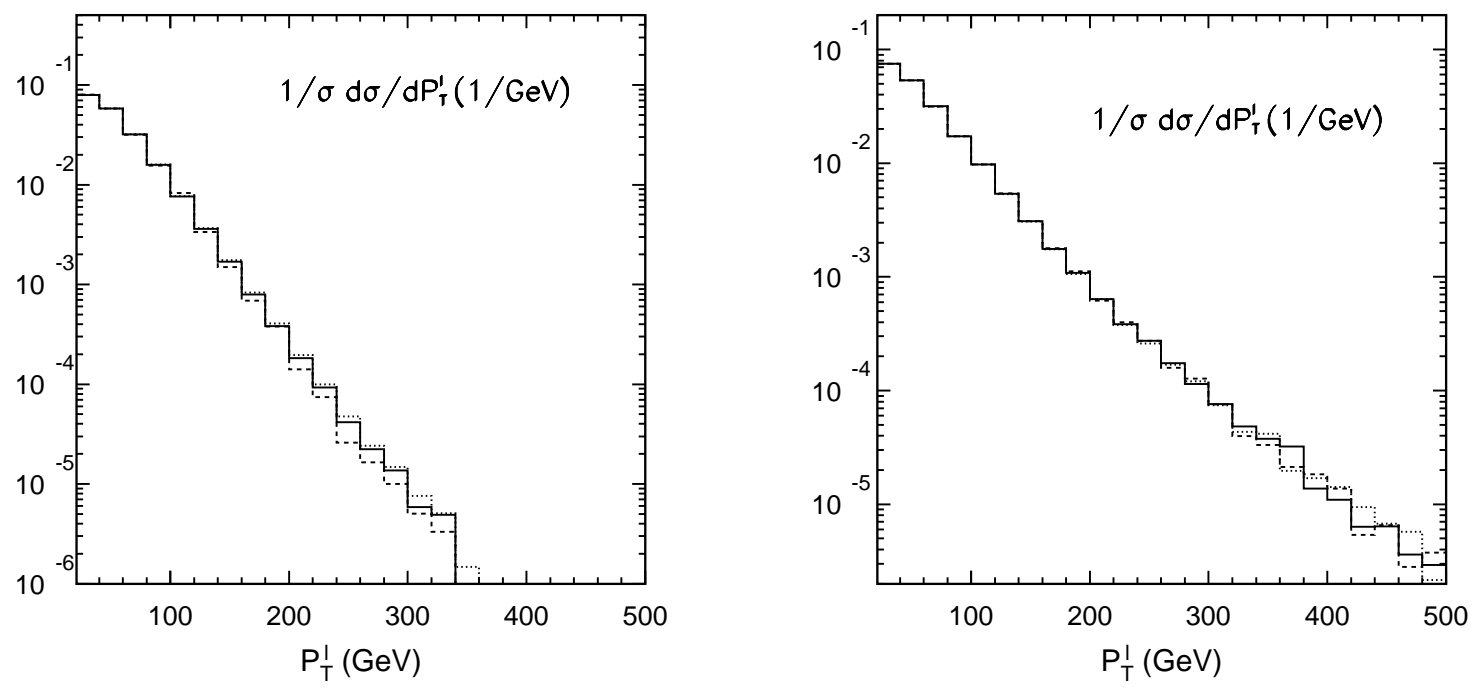

Figure 10: The normalized transverse momentum distribution of $\ell^{+}$in the $\ell \ell$ channels at NLOW, for the Tevatron (left) and the LHC at $\sqrt{s}=10 \mathrm{TeV}$ (right). The solid, dashed, and dotted lines correspond to $\mu=m_{t}, m_{t} / 2,2 m_{t}$, respectively.

$\sigma\left(t \bar{t} \rightarrow \ell^{+} \ell^{\prime-} X\right)$ in order to reduce the scale uncertainties. Nevertheless, as is well-known, the scale variations become significant at large $p_{T}^{\ell}$. Fig. 11] shows the analogous plots for the normalized $t \bar{t}$ invariant mass distribution for the semileptonic final states. The only purpose of showing these plots is to demonstrate that our code produces also established fixed order NLO QCD results. The $M_{t \bar{t}}$ distribution is, especially for the semileptonic channels, an important observable in the search for resonances that (strongly) couple to $t \bar{t}-$ for investigations within various models, cf., for instance, [96 101].

\section{IV.1. Charge asymmetries}

Next we compute, for the dilepton final states, the charge asymmetry $A$ (III.2), and the pair asymmetry $A^{t \bar{t}}$ (III.4) with the above cuts. The rapidity distributions of the charged leptons,

$$
N_{\ell^{+}}\left(y_{\ell^{+}}\right)=\frac{d \sigma}{d y_{\ell^{+}}}, \quad N_{\ell^{-}}\left(y_{\ell^{-}}\right)=\frac{d \sigma}{d y_{\ell^{-}}}
$$

are defined with polar angles of $\ell^{ \pm}$and the rapidities $y_{\ell^{ \pm}}$in the laboratory frame. They are shown in Fig. 12 (left) for the Tevatron, computed at NLOW with the cuts (IV.1). In this figure we have actually divided $N_{\ell^{ \pm}}$by the dileptonic cross section at NLOW.

One may define a differential leptonic charge asymmetry by

$$
A^{\ell}(y)=\frac{N_{\ell^{+}}(y)-N_{\ell^{-}}(y)}{N_{\ell^{+}}(y)+N_{\ell^{-}}(y)} .
$$



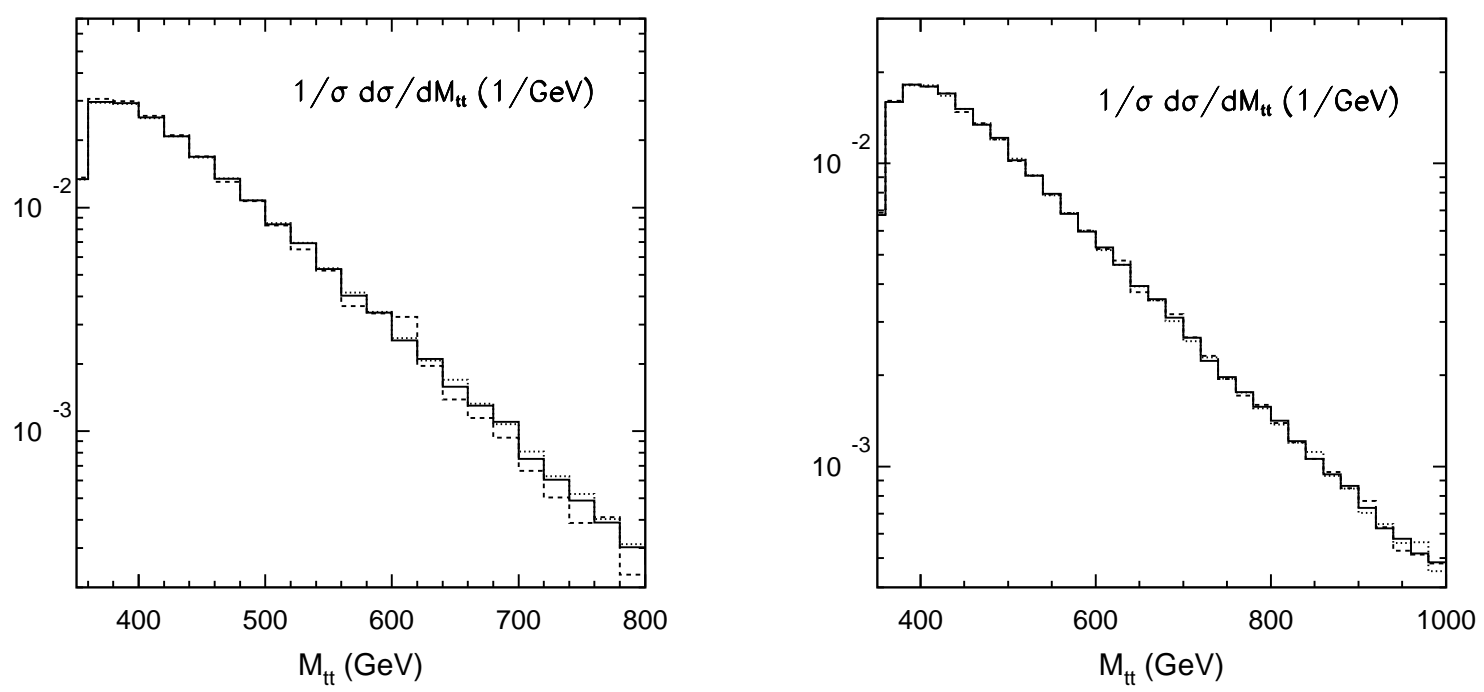

Figure 11: The normalized $t \bar{t}$ invariant mass distribution for semileptonic final states at NLOW, for the Tevatron (left) and the LHC at $\sqrt{s}=10 \mathrm{TeV}$ (right). The solid, dashed, and dotted lines correspond to $\mu=m_{t}, m_{t} / 2,2 m_{t}$, respectively.

Table 3: Results for the charge asymmetry $A$ and the pair asymmetry $A^{t \bar{t}}$ of top quarks, and the leptonic charge asymmetry $A^{\ell}$ and the pair asymmetry $A^{\ell \ell}$, for dileptonic final states at the Tevatron, with the cuts (IV.2), at NLO' and NLOW' for $\mu=m_{t} / 2, m_{t}$, and $2 m_{t}$. The acronyms NLO' and NLOW' are explained in Section III. The acronym $t \bar{t}$ uncorrelated refers to the case when all $t$ - and $\bar{t}$-spin dependent terms in the spin density matrices are switched off at NLOW.

\begin{tabular}{||c|c|c|c||c|c|c|}
\hline \hline & \multicolumn{2}{|c|}{ Tevatron $((t \bar{t}$ correlated $))$} & \multicolumn{2}{|c|}{ Tevatron $(t \bar{t}$ uncorrelated $))$} \\
\hline \hline$\mu$ & $m_{t} / 2$ & $m_{t}$ & $2 m_{t}$ & $m_{t} / 2$ & $m_{t}$ & $2 m_{t}$ \\
\hline \hline$A\left(\mathrm{NLO}^{\prime}\right)$ & 0.053 & 0.048 & 0.044 & 0.053 & 0.047 & 0.043 \\
\hline$A$ (NLOW' $\left.^{\prime}\right)$ & 0.054 & 0.049 & 0.046 & 0.054 & 0.049 & 0.046 \\
\hline \hline$A^{t t}\left(\mathrm{NLO}^{\prime}\right)$ & 0.074 & 0.068 & 0.062 & 0.075 & 0.067 & 0.061 \\
\hline$A^{t t}$ (NLOW') & 0.078 & 0.071 & 0.066 & 0.077 & 0.070 & 0.065 \\
\hline \hline$A^{\ell}\left(\mathrm{NLO}^{\prime}\right)$ & 0.038 & 0.033 & 0.031 & 0.037 & 0.033 & 0.030 \\
\hline$A^{\ell}$ (NLOW') & 0.039 & 0.034 & 0.032 & 0.038 & 0.035 & 0.032 \\
\hline$A^{\ell \ell}\left(\mathrm{NLO}^{\prime}\right)$ & 0.047 & 0.042 & 0.038 & 0.050 & 0.045 & 0.041 \\
\hline$A^{\ell \ell}\left(\mathrm{NLOW}^{\prime}\right)$ & 0.048 & 0.044 & 0.040 & 0.052 & 0.047 & 0.043 \\
\hline \hline
\end{tabular}


where $y$ denotes the rapidities $y_{\ell^{+}}$and $y_{\ell^{-}}$. This distribution is of course not identical to the differential top-quark charge asymmetry $A(y)$. It is shown in Fig. 12 (right) for the Tevatron, computed at NLOW' (i.e., by the procedure described in Sect. III) with the cuts (IV.1).

The associated leptonic charge asymmetry is

$$
A^{\ell}=\frac{\int_{y>0} N_{\ell^{+}}(y)-\int_{y>0} N_{\ell^{-}}(y)}{\int_{y>0} N_{\ell^{+}}(y)+\int_{y>0} N_{\ell^{-}}(y)} .
$$

If CP invariance holds, then $N_{\ell^{+}}\left(y_{\ell^{+}}\right)=N_{\ell^{-}}\left(-y_{\ell^{-}}\right)$and $A^{\ell}$ is equal to the leptonic forwardbackward asymmetry, $A^{\ell}=A_{F B}^{\ell^{+}}=-A_{F B}^{\ell^{-}}$, where

$$
A_{F B}^{\ell^{ \pm}}=\frac{\int_{y>0} N_{\ell^{ \pm}}(y)-\int_{y<0} N_{\ell^{ \pm}}(y)}{\int_{y>0} N_{\ell^{ \pm}}(y)+\int_{y<0} N_{\ell^{ \pm}}(y)} .
$$

In analogy to the $t \bar{t}$ pair asymmetry $A^{t \bar{t}}$ one may consider a leptonic pair asymmetry

$$
A^{\ell \ell}=\frac{\int N\left(\Delta y_{\ell}>0\right)-\int N\left(\Delta y_{\ell}<0\right)}{\int N\left(\Delta y_{\ell}>0\right)+\int N\left(\Delta y_{\ell}<0\right)},
$$

where $\Delta y_{\ell}=y_{\ell^{+}}-y_{\ell^{-}}$. The observables (IV.6), (IV.7) and related, more exclusive asymmetries were analyzed in [44] for dileptonic and semileptonic final states at the Tevatron with an approximate NLO QCD procedure (for details,see [44]). Here we compute (IV.5) and (IV.7) at NLOW' with $t, \bar{t}$ spin effects included and, for comparison, also for uncorrelated $t \bar{t}$ events. For the evaluation of $A, A^{t \bar{t}}, A^{\ell}(y), A^{\ell}$, and $A^{\ell \ell}$ we use the procedure described in the previous section. Results are given in Table 3, at NLO' and NLOW', for the dilepton final states at the Tevatron.

The distributions (IV.3) on which $A^{\ell}$ is based are displayed in Fig. 12 (left). The figure shows that the CP-symmetry relation $N_{\ell^{+}}(y)=N_{\ell^{-}}(-y)$ is satisfied. The figure displays also the consequence of the $q \bar{q}$ initiated SM top-quark charge asymmetry which tells us that the $t(\bar{t})$ is preferentially emitted into the (anti)proton direction. The numbers in Table 3 show that the charge asymmetries $A$ and $A^{t \bar{t}}$ become smaller by several percent as compared to the values given in Table 1 due to the acceptance cuts. Kinematic arguments suggest that $A^{\ell}$ has the same sign than $A$ but is smaller in magnitude, while the $\mathrm{SM}$ value of $A^{\ell \ell}$ is larger than $A^{\ell}$, in analogy to $A^{t \bar{t}}$ versus $A$. This is is corrobated by the the numbers in Table [3. We give in this table also the values of these asymmetries when all the spin-dependent terms, in particular the $t \bar{t}$ spin correlations in the matrix elements (II.4) are switched off at next-to-leading order in the gauge couplings. This corresponds to the production of uncorrelated $t \bar{t}$ pairs, followed by their decays which are spherical in the $t$ and $\bar{t}$ rest frames. One sees that the values of the inclusive asymmetries $A$ and $A^{\ell}$ remain essentially unchanged, which is not surprising. As to $A^{t \bar{t}}$, one may expect an effect of spin correlations on this ordered pair asymmetry in the presence of acceptance cuts. Yet the numbers for the correlated case are larger than those for uncorrelated events by only about $1 \%$. An inspection of the top-charge asymmetric terms in the partonic matrix elements reveals that the spin-dependent terms make indeed only a small contribution to this rather inclusive observable. 
However, as the numbers for the leptonic pair asymmetry $A^{\ell \ell}$ show, there is a moderate effect of $t \bar{t}$ spin correlations on this more exclusive observable. The difference between our predictions for correlated and uncorrelated $t \bar{t}$ events amounts to about $7 \%$.

In view of theoretical uncertainty of $\delta A \sim 30 \%$ that is implied by the analysis of [46] at the level of $t \bar{t}$ intermediate states, one may assign an uncertainty of this order of magnitude also to the numbers of Table 3. The investigation of more exclusive asymmetries at NLOW', e.g. those proposed in [44], including semileptonic $t \bar{t}$ events and the corresponding asymmetries at the LHC is beyond the scope of this paper and has to be left for a future investigation.

The D0 experiment at the Tevatron obtained, for lepton plus jets final states, a pair asymmetry $A^{t \bar{t}}=0.12 \pm 0.08 \pm 0.01$ [47]. This result must be unfolded for detector efficiencies and migration effects [47]. The pair asymmetry $A^{t \bar{t}}=0.071(7)$ given in Table 3 was computed for dileptonic final states with the above cuts, but we expect that it does not change much when computed for semileptonic events with the cuts (IV.2). With this proviso, it can be compared with the D0 result and the numbers agree within experimental and theoretical uncertainties.

The CDF collaboration measured $A^{t \bar{t}}=0.24 \pm 0.14$ [48], and the recent result of this experiment on the forward-backward asymmetry is $A_{F B}^{t}=0.193 \pm 0.065 \pm 0.024$ [49]. The CDF results are actually unfolded by using SM hypotheses, i.e. they can be compared with the predictions of Table 1. The central values of the CDF results are higher than but still consistent with the above SM expectations. The leptonic asymmetries $A^{\ell}$ and $A^{\ell \ell}$, which were predicted above at NLOW', have not yet been measured to our knowledge.

Although there is no statistically significant discrepancy between the SM expectations and the above cited experimental results, the numbers leave room for speculations about new physics contributions. One class of possible new interactions involve axial vector couplings to quarks. At the level of uncorrelated $t \bar{t}$ final states, investigations were made for instance by [45, 102].

\section{IV.2. Top-spin induced distributions and correlations}

There are a number of angular distributions and correlations with which $t, \bar{t}$ spin effects, in particular $t \bar{t}$ spin correlations can be analyzed in the $\ell \ell^{\prime}$ and $\ell+j$ final states. It is well-known that the charged lepton from top-decay is, due to the $V-A$ law (which in the meantime is known to hold for $t \rightarrow b$ to an accuracy ${ }^{7}$ of $\sim 10 \%$ [39]), the most efficient top-spin analyzer. Thus $t \bar{t}$ spin-correlation effects are most pronounced in $\ell^{+} \ell^{\prime}-$ angular correlations in the dilepton final states. A correlation of the $t$ or $\bar{t}$ spin with some axis is best analyzed by a corresponding lepton angular distribution in the semileptonic $t \bar{t}$ modes.

For qualitative reasoning below we recall here the form of the one-particle inclusive decay density matrices integrated over the energies:

$$
\begin{aligned}
& \rho^{t \rightarrow a_{1}}=\frac{\Gamma^{(1)}}{2}\left(11+\kappa_{1} \boldsymbol{\tau} \cdot \hat{\mathbf{q}}_{1}\right), \\
& \bar{\rho}^{\bar{t} \rightarrow a_{2}}=\frac{\Gamma^{(2)}}{2}\left(11-\kappa_{2} \boldsymbol{\tau} \cdot \hat{\mathbf{q}}_{2}\right),
\end{aligned}
$$

where $\hat{\mathbf{q}}_{1,2}$ are the directions of flight of $a_{1}$ and $a_{2}$ in the rest frame of the top and antitop quarks, respectively, and the $\tau^{i}$ are the Pauli matrices. As we incorporate top decay to lowest order in

\footnotetext{
${ }^{7}$ In many SM extensions new-physics contributions to the $t b W$ vertex are quite small, see e.g. [103].
} 

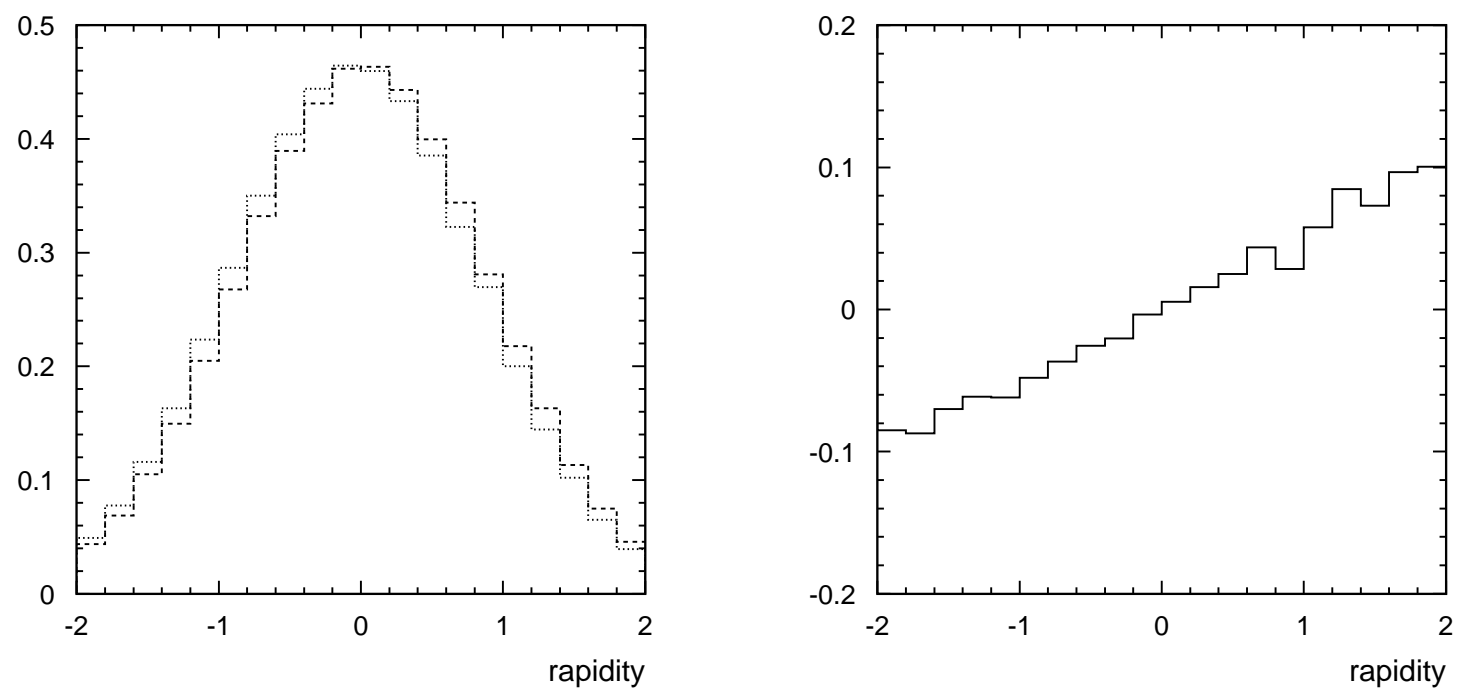

Figure 12: Left: The rapidity distributions (IV.3), normalized to $\sigma_{\ell \ell}$, for $\ell^{+}$(dashed) and $\ell^{-}$ (dotted) for the dileptonic final states at NLOW for the Tevatron for $\mu=m_{t}$. Right: The differential leptonic charge asymmetry defined in (IV.4) at NLOW' for the Tevatron for $\mu=m_{t}$.

the electroweak couplings, $\Gamma^{(2)}=\Gamma^{(1)}$ and $\kappa_{2}=\kappa_{1}$ holds to all orders in $\alpha_{s}$, if $a_{2}$ is the chargeconjugate of $a_{1}$. The spin-analyzing power of leptons is essentially one, $\kappa_{\ell^{+}}=\kappa_{\ell^{-}}=0.999$, to order $\alpha_{s}$ [33]. The spin-analyzing powers of other final state particles/jets are given to order $\alpha_{s}$ in [34].

We analyze the following correlations:

1. The double distributions:

$$
\frac{1}{\sigma} \frac{d \sigma}{d \cos \theta_{1} d \cos \theta_{2}}=\frac{1}{4}\left(1+B_{1} \cos \theta_{1}+B_{2} \cos \theta_{2}-C \cos \theta_{1} \cos \theta_{2}\right),
$$

where $\sigma$ denotes the cross section of the respective reaction. The functional form of the right-hand side holds only if cuts are not applied. Here $\theta_{1}\left(\theta_{2}\right)$ is the angle between the direction of flight of the lepton $\ell^{+}$or jet $j_{1}\left(\ell^{\prime-}\right.$ or $\left.j_{2}\right)$ from $t(\bar{t})$ decay in the $t(\bar{t})$ rest frame and a reference direction $\hat{\mathbf{a}}(\hat{\mathbf{b}})$. Below we will choose the axes defined in the last section. The coefficient $C$ is a measure of $t \bar{t}$ spin correlations - it is induced by the correlation (III.5). In the absence of cuts we have the formula [13]:

$$
\left.C=\kappa_{1} \kappa_{2}\left\langle 4 \mathbf{S}_{t} \cdot \hat{\mathbf{a}}\right)\left(\mathbf{S}_{\bar{t}} \cdot \hat{\mathbf{b}}\right)\right\rangle,
$$

which holds for factorizable corrections to all order 8 . The expectation values on the righthand side of this equation are given in Table 2. The coefficients $B_{1,2}$ will be discussed in items 4 and 5 .

\footnotetext{
${ }^{8}$ In fact, the nonfactorizable $\mathcal{O}\left(\alpha_{s}^{3}\right)$ QCD corrections [26] do not change this result; cf. the comment in [14].
} 
2. The opening angle distribution [14]

$$
\frac{1}{\sigma} \frac{d \sigma}{d \cos \varphi}=\frac{1}{2}(1-D \cos \varphi)
$$

where $\varphi$ denotes the angle between the direction of flight of the lepton $\ell^{+}$(or jet $j_{1}$ ) and of $\ell^{\prime-}$ (or $j_{2}$ ), defined in the $t$ and $\bar{t}$ rest frame, respectively. The functional form of the right-hand side applies only in the absence of cuts. The coefficient $D$ is induced by a term proportional to $\mathbf{S}_{t} \cdot \mathbf{S}_{\bar{t}}$ in the $t \bar{t}$ production density matrix. If no cuts are applied the formula

$$
D=\kappa_{1} \kappa_{2}\left\langle\frac{4}{3} \mathbf{S}_{t} \cdot \mathbf{S}_{\bar{t}}\right\rangle
$$

holds. The expectation values on the right-hand side of this equation are given in Table 2 , Below we comment also on the leptonic opening angle distribution in the laboratory frame.

3. The dileptonic azimuthal angle correlation, $\sigma^{-1} d \sigma / d \Delta \phi,[51,53$. Here $\Delta \phi$ is the difference of the azimuthal angles of the $\ell^{+}$and $\ell^{\prime-}$ directions of flight, which can be measured in the laboratory frame. This distribution was recently analyzed by [64] for the case of dileptonic $t \bar{t}$ events at the LHC. It was shown by these authors that the $\Delta \phi$ distribution is sensitive to SM spin correlations if only events with $M_{t \bar{t}}<400 \mathrm{GeV}$ are taken into account. The price one pays for excluding with this cut a large fraction of the dileptonic events is balanced by the fact that the $\Delta \phi$ distribution is easier to measure in the dilepton channels than (IV.10) or (IV.12).

4. Nonzero coefficients $B_{1}$ and $B_{2}(\underline{I V .10})$ would be due to a correlation $\mathbf{S}_{t} \cdot \hat{\mathbf{a}}$ and $\mathbf{S}_{\bar{t}} \cdot \hat{\mathbf{b}}$ of the $t$ and $\bar{t}$ spin with the respective axis. If polar vectors $\hat{\mathbf{a}}, \hat{\mathbf{b}}$ are chosen then $B_{i} \neq 0$ would signal parity violation if no cuts were made. For the axes defined in Section III the weak-interaction corrections induce non-zero, albeit very small $B_{i}$ [20,21]. If cuts are not applied one obtains from (IV.10):

$$
\frac{1}{\sigma} \frac{d \sigma}{d \cos \theta_{1,2}}=\frac{1}{2}\left(1+B_{1,2} \cos \theta_{1,2}\right)
$$

In view of the very small parity-violating effects just mentioned, the SM predicts this distribution to be essentially flat [21] if cuts could be applied that are parity-symmetric with respect to the $t$, respectively $\bar{t}$ rest frame. However, realistic cuts severely distort (IV.14). We compute for the semileptonic $t \bar{t}$ modes at the LHC:

$$
G_{\ell^{+}}\left(z_{+}\right)=\frac{1}{\sigma_{\ell^{+}}} \frac{d \sigma}{d z_{+}}, \quad G_{\ell^{-}}\left(z_{-}\right)=\frac{1}{\sigma_{\ell^{-}}} \frac{d \sigma}{d z_{-}}
$$

where $z_{ \pm}=\cos \theta_{\ell^{ \pm}}$and $\cos \theta_{\ell^{+}}=\hat{\mathbf{a}} \cdot \hat{\ell}^{+}, \cos \theta_{\ell^{-}}=\hat{\mathbf{b}} \cdot \hat{\ell}^{-}$. Below we use for definiteness the helicity basis. One can nevertheless use these distributions for parity and also for CP tests, cf. the comments at the end of this section. 
5. The functional forms of the r.h.s. of Eqs. (IV.10), (IV.12), and (IV.14) do not hold in the presence of kinematic cuts. Kinematic considerations make it clear that the forms (IV.10) and (IV.14) will be stronger distorted than (IV.12). Nevertheless, the right-hand side of (IV.10 $)$ - or of (IV.12 $)$ - may be used in fits to data for determining the correlation coefficients $C$ or $D$. One may also divide the events in an experimental analysis into spinenhanced (low $M_{t \bar{t}}$ ) and spin-depleted (high $M_{t \bar{t}}$ ) samples and form ratios in order to reduce systematic uncertainities [104].

Here we use the estimators [14]:

$$
\begin{array}{rr}
\hat{C}=-9\left\langle\cos \theta_{1} \cos \theta_{2}\right\rangle, & \hat{D}=-3\langle\cos \varphi\rangle, \\
\hat{B}_{1}=3\left\langle\cos \theta_{1}\right\rangle, & \hat{B}_{2}=3\left\langle\cos \theta_{2}\right\rangle .
\end{array}
$$

In the absence of cuts, $\hat{C}=C, \hat{D}=D, \hat{B}_{i}=B_{i}$. Below we compute the expectation values (IV.16) with the above cuts. We drop the hats from now on for ease of notation. In fact, we compute the variation of the correlations with $M_{t \bar{t}}$. We define it in the case of $D$ by

$$
\frac{d D}{d M_{t \bar{t}}}=\frac{-3}{\sigma} \int d \sigma \cos \varphi \delta\left(\sqrt{\left(\sum p_{f}\right)^{2}}-M_{t \bar{t}}\right) \Theta_{\mathrm{cut}},
$$

Here $\Theta_{\text {cut }}$ denotes the product of theta functions implementing the cuts and, in case of an exclusive computation, of jet functions. For the other angular variables in (IV.16) the variations are defined in analogous fashion. We normalize to the respective integrated cross section. The computations of (IV.17) and of the other observables concerning their variation with respect to $M_{t \bar{t}}$ made in this paper are theory predictions; i.e. the complications in the actual measurement of the invariant mass of the $t \bar{t}$ pair are not taken into account; cf. the comments below.

Fig. 13 shows $d C_{\text {beam }} / d M_{t \bar{t}}$ and the opening angle distribution (IV.12) for the Tevatron for three scales. The resulting integrated correlation coefficients are given in Table 4. Comparing $C_{\text {beam }}=0.614(10)$ with the "no cut" value $0.791(14)$ shows the significant effects of the cuts on this correlation. The distribution $d C_{\text {off }} / d M_{t \bar{t}}$, which is not shown here, has essentially the same form as $d C_{\text {beam }} / d M_{t \bar{t}}$. The correlation coefficient $C_{\text {off }}$ in the off-diagonal basis is also reduced by the cuts to $C_{\text {off }}=0.621(10)$, cf. Table 4. Without cuts $C_{\text {off }}=0.798(15)$.

The correlations can be understood qualitatively in terms of simple spin arguments. We recall this here only for the opening angle distribution, using (IV.13) and Born level considerations. The $t \bar{t}$ pair produced by the dominant LO reaction $q \bar{q} \rightarrow t \bar{t}$ is in a ${ }^{3} S_{1}$ state near threshold. Thus, $\left\langle\mathbf{S}_{t} \cdot \mathbf{S}_{\bar{t}}\right\rangle_{q \bar{q}}=1 / 4$ and $D_{q \bar{q}}=1 / 3$. The "true" $t \bar{t}$ intermediate state is of course a mixed state. The component from $g g$ fusion, the radiative corrections, and the cuts dilute this correlation to $D=0.145(6)$, cf. Table 4. Comparing with the "no cut" value 0.218(2) shows again the significant effect of the cuts.

On may attempt to optimize these correlation coefficients by applying an additional cut on $M_{t \bar{t}}$, but in view of the not very large number of dileptonic $t \bar{t}$ events at the Tevatron this seems not very useful. In Table 4 we give the values of several correlation coefficients for dileptonic events at the Tevatron with $M_{\max }=550 \mathrm{GeV}$. This value has been chosen here rather arbitrarily; in fact, 
the correlations do not increase when this cut is tightened to $400 \mathrm{GeV}$. With even tighter cuts one would discard too much dileptonic events.

Figs. 14, 15, and 16] show $d C_{\text {hel }} / d M_{t \bar{t}}$, the opening angle distribution (IV.12), and $d D / d M_{t \bar{t}}$ for dileptonic final states at the LHC with $\sqrt{s}=10$ and $14 \mathrm{TeV}$. The distribution $d C_{\text {hel }} / d M_{t \bar{t}}$ changes sign around $M_{t \bar{t}} \sim 700 \mathrm{GeV}$. This is because for values of $M_{t \bar{t}}$ not too far above threshold, the QCD dynamics tells us that the number of $t \bar{t}$ pairs produced with like helicities is larger than those with unlike helicities, while at large $M_{t \bar{t}}$ helicity conservation of the strong and weak interactions implies that it is the other way around. As the event numbers become small very rapidly and because we have normalized to $\sigma_{\ell \ell}$, the distribution $d C_{\text {hel }} / d M_{t \bar{t}}$ displayed in Figs. 14 and 15 is essentially zero above $M_{t \bar{t}} \simeq 700 \mathrm{GeV}$.

As to the qualitative understanding of the opening angle distribution, Figs. 14] and 15: Because the production of $t \bar{t}$ pairs at the LHC is dominated by gluon fusion, the pair is, to LO QCD, in a ${ }^{1} S_{0}$ state at threshold. Hence $\left\langle\mathbf{S}_{t} \cdot \mathbf{S}_{\bar{t}}\right\rangle_{g g}=-3 / 4$ and $D_{g g}=-1$. Away from threshold, the pair is actually in a mixed state already at Born level. This fact, the component from $q \bar{q}$ annihilation, and the radiative corrections deplete this correlation to $D=-0.252(5)(10 \mathrm{TeV})$, respectively $D=-0.240(10)(14 \mathrm{TeV})$, cf. Table 4 . The effect of cuts on $D$ is less drastic than in the case of the Tevatron, as seen by comparing with the "no cut" values -0.233(4) (10 TeV) and $-0.236(3)(14 \mathrm{TeV})$. On the other hand, the helicity correlation is depleted by the cuts, as shown by comparing the respective numbers in Table 4 with $C_{\text {hel }}$ (no cut) $=0.326(2)(10 \mathrm{TeV})$ and $0.328(3)(14 \mathrm{TeV})$.

The helicity and opening angle correlations at the LHC can be enhanced significantly by taking into account only events with a $t \bar{t}$ invariant mass below a certain value $M_{\max }$. Table 4 shows that for $M_{\max }=550 \mathrm{GeV}$ the correlation coefficients $D$ and $C_{\text {hel }}$ can be enhanced by about $43 \%$ and $50 \%$, respectively. We have not optimized this cut.

The beam, off-diagonal, and helicity correlation and the opening angle distribution were computed before to NLO QCD for dileptonic, semileptonic, and non-leptonic final states in [14], without cuts, and with a different set of PDF (CTEQ6.1M and MRST2003) and a slightly different value of the top-quark mass. The numbers given in that paper can be compared with the results given here, using Table 2, the formulae (IV.11) and (IV.13), and the spin-analyzing powers $\kappa$ given in [14]. There are two effects: the PDF set CTEQ6.6M and the smaller value of the top-quark mass that we use in the present paper enhances the correlation coefficients slightly as compared to those of [14]. On the other hand the weak-interaction corrections lead to a slight reduction of the correlations at the LHC (cf. Sect. III), while their influence on the Tevatron correlations is smaller. In effect this leads to slightly enhanced numbers for the Tevatron, about $2 \%$, while the numbers for the LHC given in Table 2 are essentially the same as those in [14.

The authors of [88] computed the opening angle distribution as defined above in the MC@NLO framework - whith parton showering, but no cuts were applied - for dileptonic final states at the Tevatron and the LHC (14 TeV) and found agreement with the results given in [14]. This shows that this leptonic correlation is essentially unaffected by the parton shower. This distribution was recently also evaluated in [91] for dileptonic final states at the Tevatron and the LHC (10 TeV), to NLO QCD with cuts. We have computed these distributions also with the selection criteria of [91] and agree with their results. 

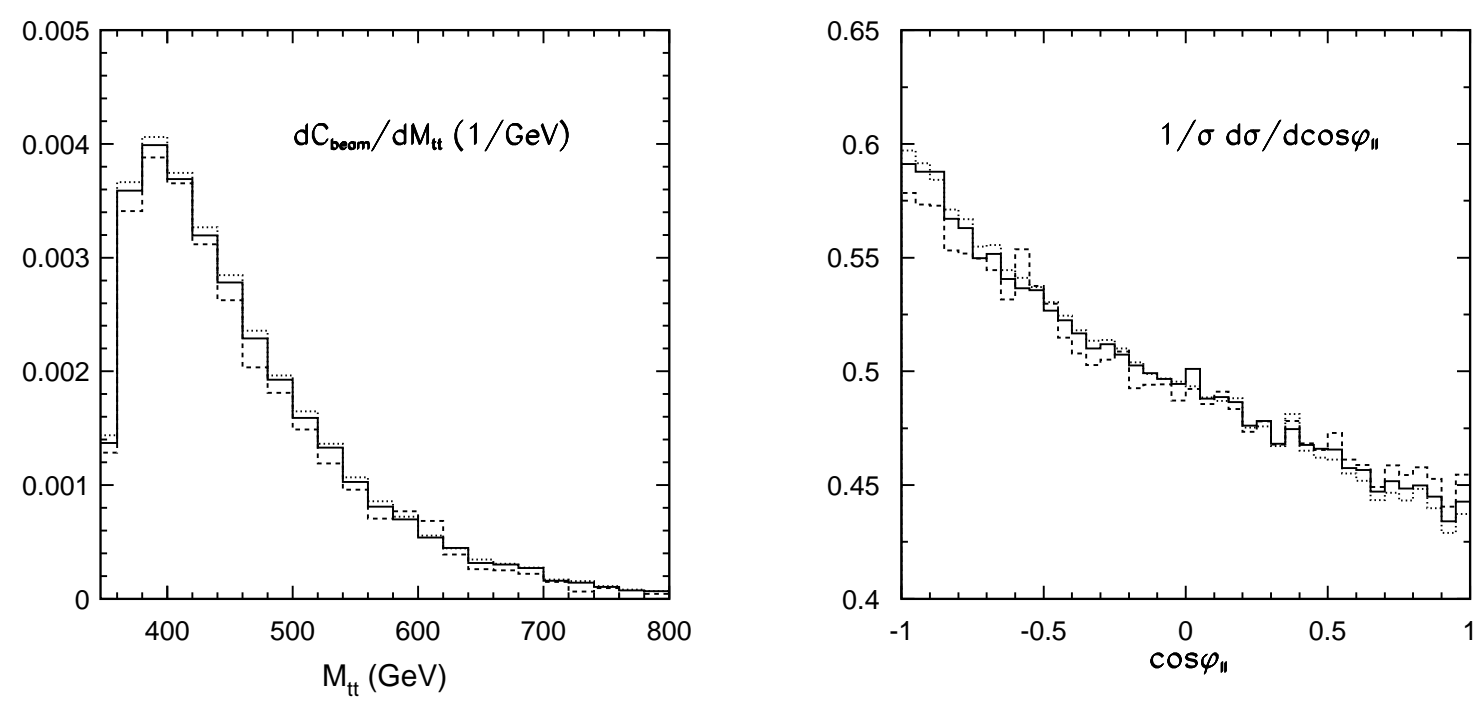

Figure 13: Distributions at NLOW for $\ell \ell$ final states at the Tevatron: $d C_{\text {beam }} / d M_{t \bar{t}}$ (left panel) and the opening angle distribution (IV.12) (right panel). The solid, dashed, and dotted lines correspond to $\mu=m_{t}, m_{t} / 2$, and $2 m_{t}$, respectively.

The dileptonic angular correlations considered above require the reconstruction of the $t$ and $\bar{t}$ rest frames, which is a highly non-trivial experimental task. There are enough kinematic constraints but, as is well-known, for dileptonic events a four-fold ambiguity arises, which may increase to an eightfold one if the $b$ and $\bar{b}$ jets are incorrectly assigned. Monte-Carlo based methods have been devised (see, e.g., [105]) in order to reduce or partly resolve these ambiguities.

The beam-basis and off-diagonal correlations were recently measured for dileptonic events at the Tevatron by the D0 and CDF collaborations, respectively, with the results

$$
C_{\text {beam }}=-0.17_{-0.53}^{+0.64} \quad[65], \quad \kappa_{\text {off }}=0.32_{-0.78}^{+0.55} \quad[66] .
$$

In our sign convention $\kappa_{\text {off }}=C_{\text {off }}$. These results agree, within the still large experimental uncertainties, with our predictions for the dilepton channels at the Tevatron given Table 4. The CDF collaboration measured also the helicity correlation for lepton + jets events. They obtained for the respective spin correlation parameter at the $t \bar{t}$ level the value $\kappa_{\text {hel }}=0.60 \pm 0.50 \pm 0.16$ [67]. In our sign convention for the double spin asymmetries [14] this is equivalent to $-\mathcal{O}_{\text {hel }}$ and should be compared with the value $-\mathcal{O}_{\text {hel }}=+0.368(10)$ for the Tevatron given in Table 2 . The above acceptance cuts deplete this value to $-C_{\text {hel }}=+0.30(6)$, cf. Table 4 .

A simulation study [106] within the ATLAS collaboration concluded that $D$ and $C_{\text {hel }}$ can be measured at the LHC with a relative uncertainty of $\delta D \simeq 5 \%$ and $\delta C_{\text {hel }} \simeq 7 \%$, respectively, using dileptonic and semileptonic $t \bar{t}$ events. 

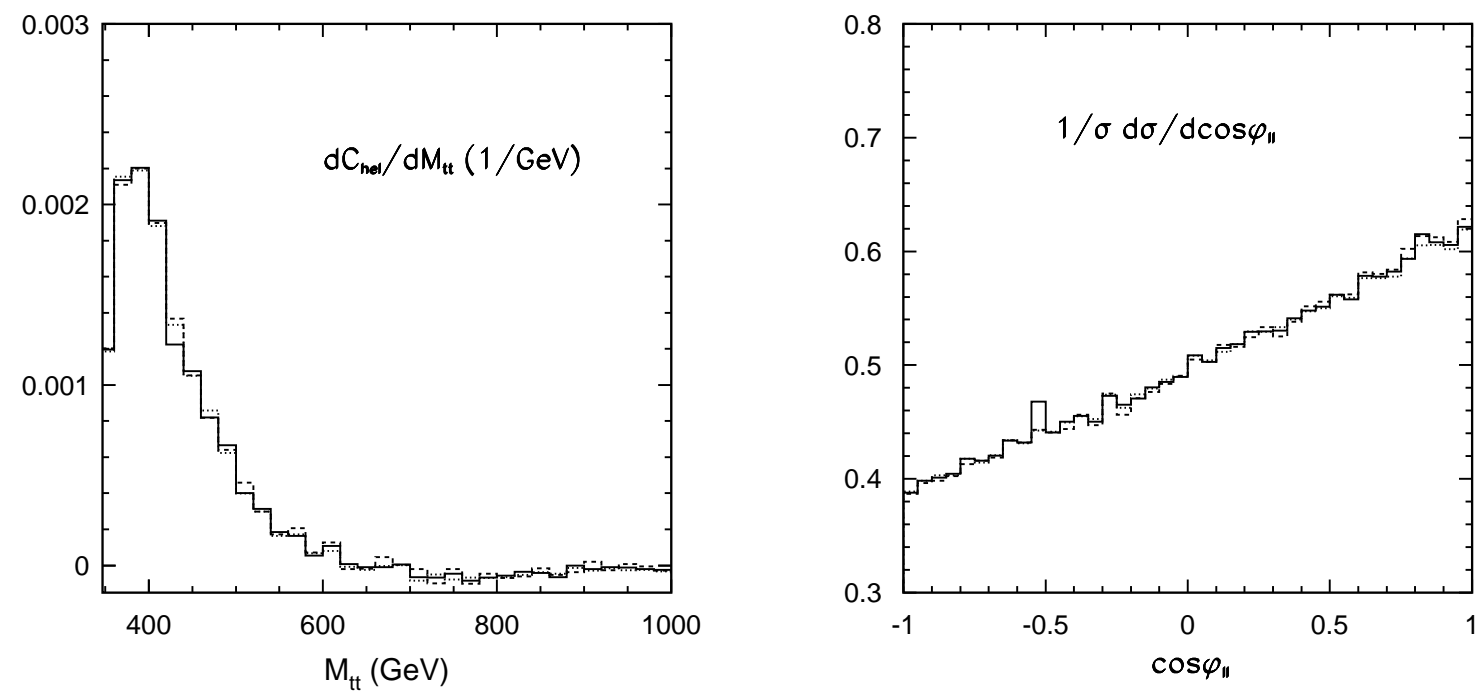

Figure 14: Distributions at NLOW for $\ell \ell$ final states at the LHC $(\sqrt{s}=10 \mathrm{TeV}): d C_{\mathrm{hel}} / d M_{t \bar{t}}(\mathrm{left}$ panel) and the opening angle distribution (IV.12) (right panel). The solid, dashed, and dotted lines correspond to $\mu=m_{t}, m_{t} / 2$, and $2 m_{t}$, respectively.
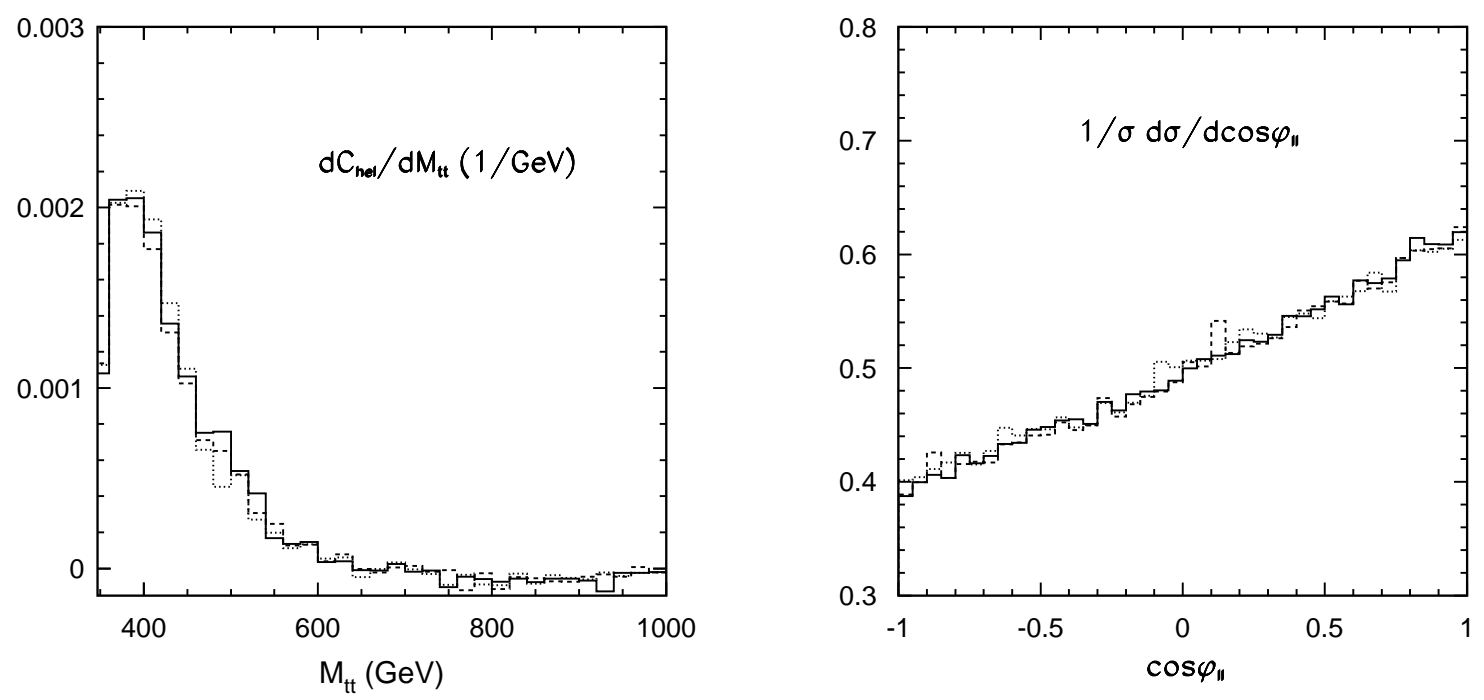

Figure 15: Distributions at NLOW for $\ell \ell$ final states at the LHC $(\sqrt{s}=14 \mathrm{TeV}): d C_{\text {hel }} / d M_{t \bar{t}}($ left panel) and the opening angle distribution (IV.12) (right panel). The solid, dashed, and dotted lines correspond to $\mu=m_{t}, m_{t} / 2$, and $2 m_{t}$, respectively. 

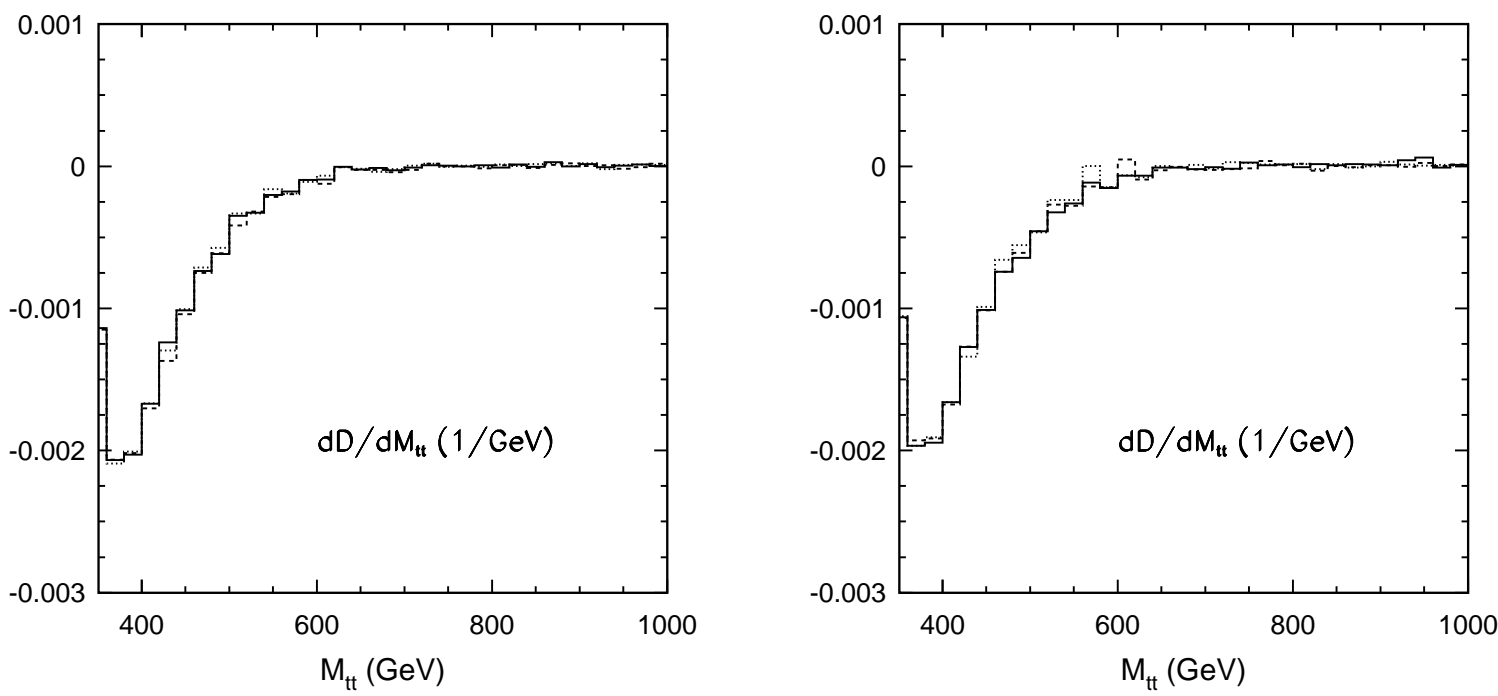

Figure 16: The distribution $d D / d M_{t \bar{t}}$ at NLOW for $\ell \ell$ final states at the LHC at $\sqrt{s}=10 \mathrm{TeV}$ (left panel) and at $14 \mathrm{TeV}$ (right panel). The solid, dashed, and dotted lines correspond to $\mu=m_{t}, m_{t} / 2$, and $2 m_{t}$, respectively.

Table 4: Results for several observables at NLOW for dileptonic final states at the Tevatron and LHC, with cuts as specified in (IV.11). The correlation coefficients are computed also with a cut $M_{t \bar{t}}<M_{\max }$. Here we have chosen $M_{\max }=550 \mathrm{GeV}$.

\begin{tabular}{||c|c|c|c||c|c|c||c|c|c||}
\hline \hline & \multicolumn{3}{|c|}{ Tevatron } & \multicolumn{3}{c|}{ LHC $(10 \mathrm{TeV})$} & \multicolumn{3}{c||}{ LHC $(14 \mathrm{TeV})$} \\
\hline \hline$\mu$ & $m_{t} / 2$ & $m_{t}$ & $2 m_{t}$ & $m_{t} / 2$ & $m_{t}$ & $2 m_{t}$ & $m_{t} / 2$ & $m$ & $2 m$ \\
\hline \hline$\sigma_{\ell \ell}(\mathrm{pb})$ & 0.043 & 0.042 & 0.038 & 2.31 & 2.03 & 1.76 & 5.00 & 4.38 & 3.82 \\
\hline \hline$D$ & 0.139 & 0.145 & 0.151 & -0.257 & -0.252 & -0.257 & -0.240 & -0.247 & -0.230 \\
\hline$D\left(M_{\max }\right)$ & 0.125 & 0.132 & 0.138 & -0.344 & -0.340 & -0.347 & -0.340 & -0.353 & -0.338 \\
\hline \hline$C_{\text {hel }}$ & -0.294 & -0.299 & -0.306 & 0.249 & 0.247 & 0.252 & 0.225 & 0.237 & 0.229 \\
\hline$C_{\text {hel }}\left(M_{\max }\right)$ & -0.256 & -0.262 & -0.269 & 0.350 & 0.351 & 0.362 & 0.336 & 0.360 & 0.345 \\
\hline \hline$B_{1}$ & & & & 0.174 & 0.176 & 0.174 & 0.162 & 0.162 & 0.178 \\
\hline \hline$C_{\text {beam }}$ & 0.605 & 0.614 & 0.624 & & & & & & \\
\hline$C_{\text {beam }}\left(M_{\max }\right)$ & 0.577 & 0.586 & 0.596 & & & & & & \\
\hline$C_{\text {off }}$ & 0.612 & 0.621 & 0.631 & & & & & & \\
\hline \hline$C_{\text {off }}\left(M_{\text {max }}\right)$ & 0.582 & 0.591 & 0.601 & & & & & & \\
\hline \hline
\end{tabular}


Next we analyze two leptonic angular correlations defined in the laboratory frame. The distribution of the opening angle between the lepton momenta in the laboratory frame, $\sigma^{-1} d \sigma / d \cos \varphi^{L}$, which was recently considered at NLO QCD in [91, is almost insensitive to $t \bar{t}$ spin correlations 96 . This is demonstrated in Fig. 17 (left panel) where this distribution is shown for the LHC with9 and without $t \bar{t}$ spin correlations. The correlation of the boosted leptons in the laboratory frame traces the correlation of the $t$ and $\bar{t}$ spins inefficiently. On the other hand, the distribution (IV.12) discriminates by construction 10 between spin correlations "switched-on" and "switched-off", cf. Fig. 17 (right panel).

As shown recently [64] by a tree-level analysis, the dileptonic azimuthal angle correlation at the LHC, $\sigma^{-1} d \sigma / d \Delta \phi$, does discriminate between correlated and uncorrelated $t \bar{t}$ events if dileptonic final states with $M_{t \bar{t}}<400 \mathrm{GeV}$ are taken into account only. Here $\Delta \phi$ is the difference of the azimuthal angles of $\ell^{+}$and $\ell^{\prime-}$ in the laboratory frame. This distribution is plotted in Fig. 18 at LO and NLOW for correlated and uncorrelated dileptonic $t \bar{t}$ events. In [64] only the LO distribution is given with which we agree. For the cross section ratio $r=\sigma_{\ell \ell}\left(M_{t \bar{t}}<400 \mathrm{GeV}\right) / \sigma_{\ell \ell}$ we get with the above cuts $r \simeq 18.6 \%$ at NLO QCD with $\mu=m_{t}$. Assuming an integrated luminosity of 10 $\mathrm{fb}^{-1}$ at the LHC $(14 \mathrm{TeV})$, this corresponds to $\sim 32000$ dilepton events $(\ell=e, \mu)$.

The shapes of the correlated and uncorrelated distribution depend very sensitively on the cut on $M_{t \bar{t}}$. Thus, a critical (experimental) issue will be how well the theory-cut on $M_{t \bar{t}}$ can be realized in the selection of dileptonic events at the LHC, given the ambiguities that arise from the presence of the two neutrinos in the final state. This complication was analyzed in [64]. In addition, the azimuthal angle correlation looses its discriminating power the more the cut on $M_{t \bar{t}}$ is relaxed to values above $M_{\max }=400 \mathrm{GeV}$. Fig. 19 shows this distribution at NLOW for correlated and uncorrelated dileptonic $t \bar{t}$ events (with cuts as given in (IV.1)) if no cut on $M_{t \bar{t}}$ is applied. The plot compares well with Fig. 1 of [88] computed with the MC@NLO code. (The above comments on the differences between [88] and our approach in the computation of the openening angle distribution apply also here.)

The azimuthal angle correlation is clearly easier and more precisely measurable (assuming that the problem of finding the best option for selecting dileptonic events will be solved) than the helicity correlation or the opening angle distribution (IV.12). It can be used to check the SM prediction on $t \bar{t}$ spin correlations or may be employed in the search for new physics effects in the low energy tail of the $M_{t \bar{t}}$ spectrum. If one one wants to keep spin correlation observables in the tool kit, especially for new physics searches in the upper part of the $M_{t \bar{t}}$ spectrum, where new resonances or continuum effects may show up, one should use observables like the helicity correlation or the opening angle distribution.

The beam, off-diagonal, and helicity correlation and the opening angle distribution (IV.12) can also be measured for semileptonic $t \bar{t}$ events at the Tevatron and at the LHC. In this case the $t$ and $\bar{t}$ rest frames are easier and more efficiently reconstructible than for dileptonic final states. Moreover the number of $\ell+$ jets events is about six times larger than the number of $\ell \ell^{\prime}$ events. In the case of a hadronically decaying top quark the least energetic non- $b$ jet $j<$ among the top-decay products

\footnotetext{
${ }^{9}$ For comparison we have computed this distribution also with the cuts used in [91] and find agreement with Fig. 4 of this work.

${ }^{10}$ This is of course also the case for the beam, off-diagonal, and helicity correlations.
} 

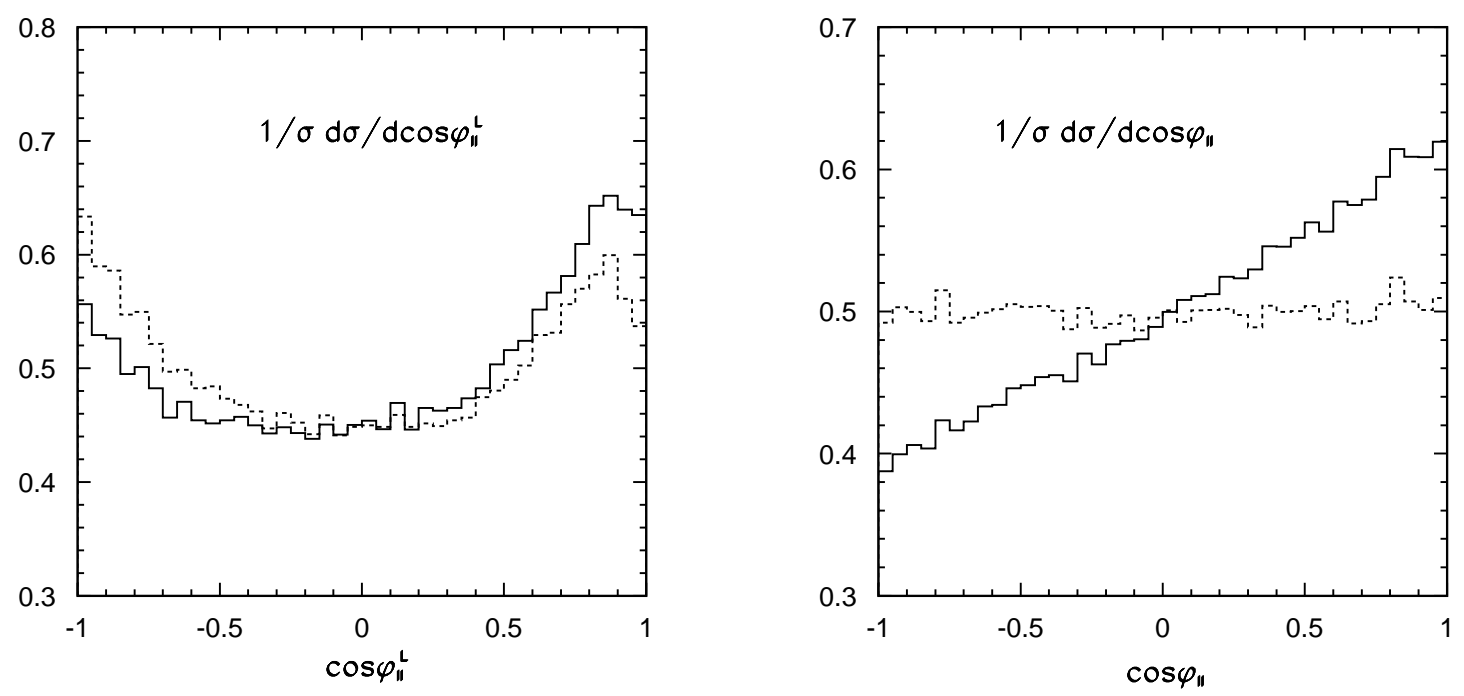

Figure 17: Left: The opening angle distribution $\sigma^{-1} d \sigma / d \cos \varphi^{L}$ in the laboratory frame. Right: The opening angle (IV.12) defined with respect to the $t \bar{t}$ ZMF. Both are computed at NLOW with $\mu=m_{t}$ for $\ell \ell$ final states at the $\operatorname{LHC}(\sqrt{s}=14 \mathrm{TeV})$. The solid lines are the SM predictions, the dashed lines are the distributions which result when the $t \bar{t}$ spin correlations are switched off.

may serve as analyzer of the top spin. It was shown [34 that $j_{<}$has the best spin-analyzing power for this decay mode (barring the extremely difficult task of tagging the flavor of the $d$-type jet from $W$ decay); $\kappa_{j_{<}} \simeq 0.47$ to order $\alpha_{s}$ depending on the jet algorithm. Thus the angular correlations for the $\ell+$ jets events will be reduced roughly by this factor as compared with the respective correlations for $\ell \ell^{\prime}$ final states. Detailed predictions with acceptance cuts will be given elsewhere [107].

Finally we consider, for the the $\ell^{ \pm}+$jets modes, the one-particle distributions $G_{\ell^{ \pm}}\left(z_{ \pm}\right)$defined in (IV.15) where $z_{ \pm}=\cos \theta_{\ell^{ \pm}}$denote the cosines of the lepton helicity angles, i.e., $\theta_{\ell^{+}}\left(\theta_{\ell^{-}}\right)$is the angle between the $\ell^{+}\left(\ell^{-}\right)$direction of flight in the $t(\bar{t})$ rest frame and the $t(\bar{t})$ direction in the $t \bar{t} \mathrm{ZMF}$.

If no cuts are applied, non-trivial, i.e. non-flat distributions would arise if the ensemble of (anti)top quarks were longitudinally polarized - a small effect in the SM [20,21]. However, the presence of cuts severely distort these distributions in the vicinity of $\cos \theta_{\ell^{ \pm}}=-1$. This region corresponds to leptons that are emitted into the hemisphere opposite to the (anti)top direction of flight in the $t \bar{t}$ ZMF. These leptons are therefore less energetic on average and thus stronger affected by cuts than those in the remaining region. This is shown in Fig. 20 for $G_{\ell^{+}}$and the corresponding differential distribution $d B_{1} / d M_{t \bar{t}}$ at the LHC. The SM value of $B_{1}$ is given, for the above cuts, in Table 4, To NLO in the gauge couplings, the distributions $G_{\ell^{-}}$and $d B_{2} / d M_{t \bar{t}}$ are identical to $G_{\ell^{+}}$and $d B_{1} / d M_{t \bar{t}}$, respectively, which we checked with our code. This is because

$$
G_{\ell^{+}}\left(z_{+}\right)=G_{\ell^{-}}\left(z_{-}\right)
$$



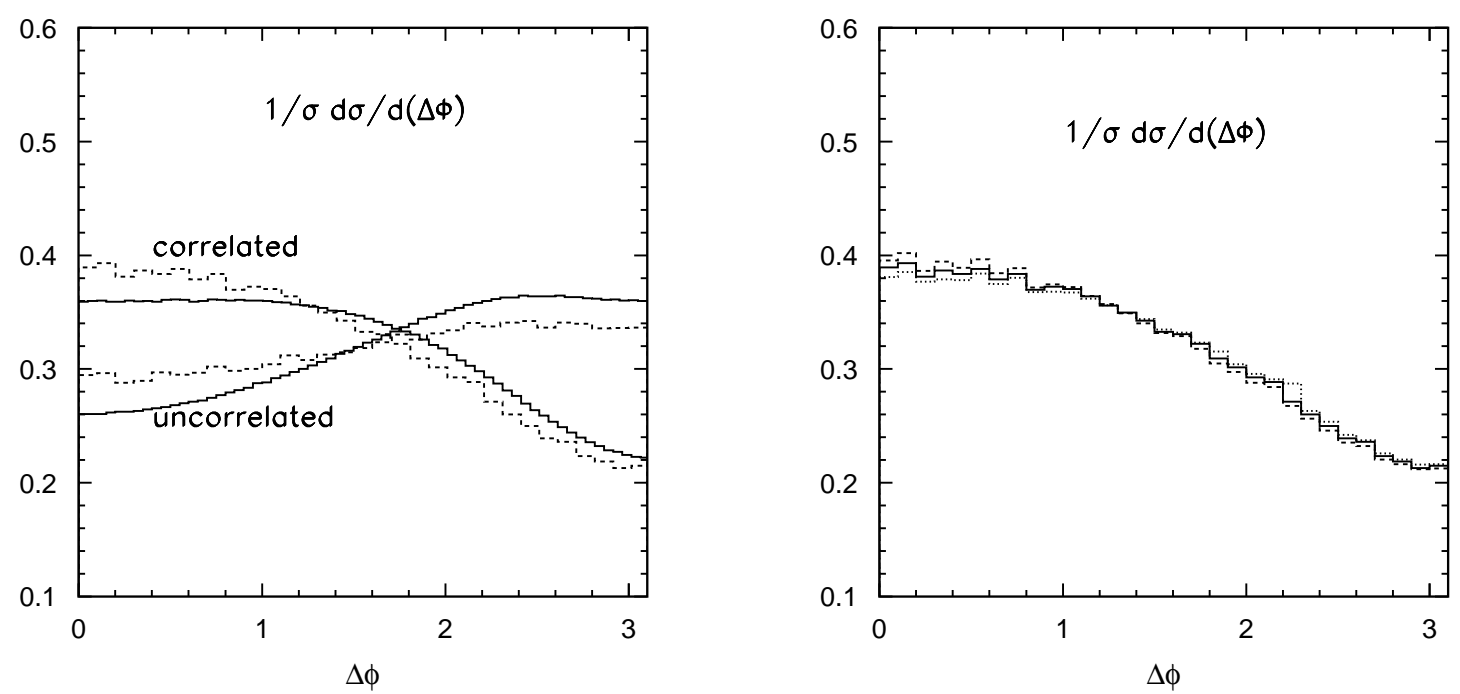

Figure 18: The azimuthal angle distribution $\sigma^{-1}\left(M_{\max }=400 \mathrm{GeV}\right) d \sigma / d \Delta \phi$ in the laboratory frame for $\ell \ell$ final states at the LHC $(14 \mathrm{TeV})$ with and without $t \bar{t}$ spin correlations, with the additional cut $M_{t \bar{t}}<M_{\max }$. Solid and dashed lines are LO and NLOW, respectively, for $\mu=m_{t}$. Right: The distribution at NLOW for $\mu=m_{t}$ (solid), $m_{t} / 2$ (dashed), and $2 m_{t}$ (dotted).

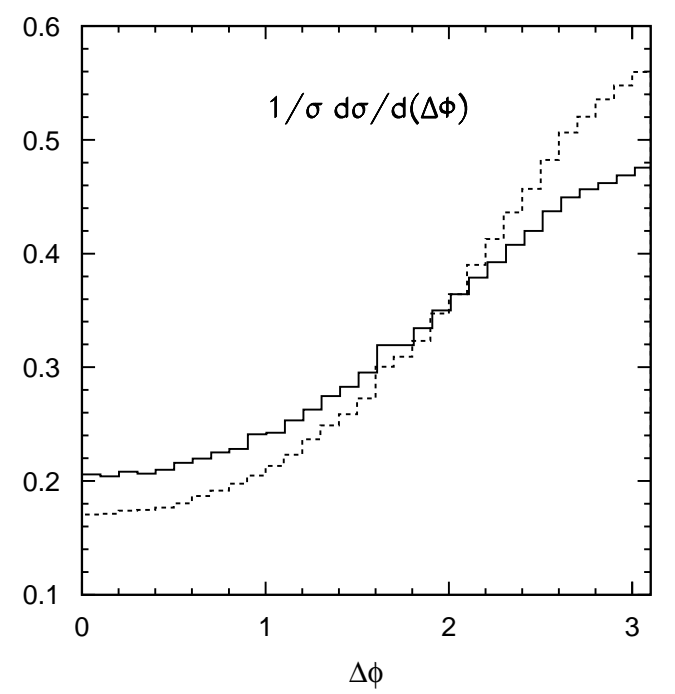

Figure 19: Left: The azimuthal angle distribution $\sigma^{-1} d \sigma / d \Delta \phi$ in the laboratory frame for $\ell \ell$ final states at the LHC $(14 \mathrm{TeV})$ at NLOW for $\mu=m_{t}$ and no cut on $M_{t \bar{t}}$. The solid line is with and the dashed line without $t \bar{t}$ spin correlations. 

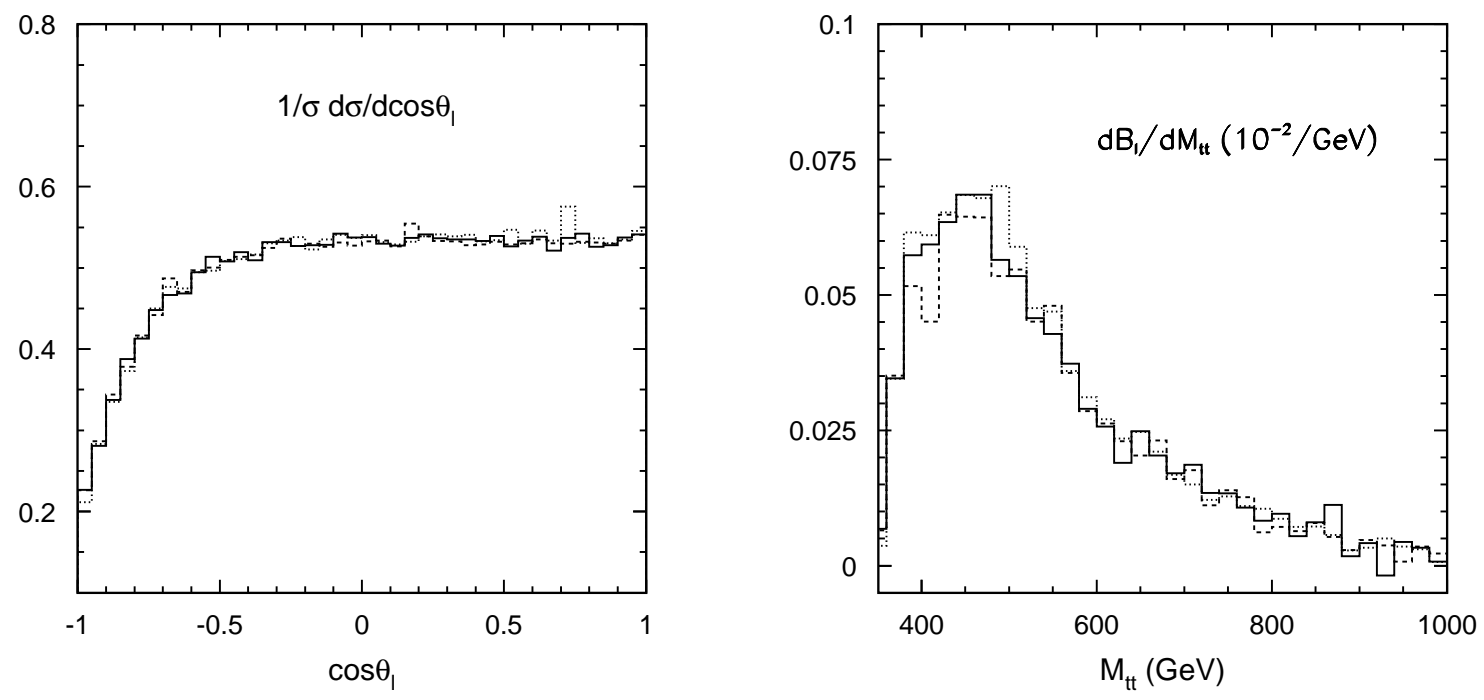

Figure 20: The distribution $\sigma^{-1} d \sigma / d \cos \theta_{\ell^{+}}$(left panel) and the corresponding differential distribution $d B_{1} / d M_{t \bar{t}}$ (right panel) for $\ell^{+}+$jets at the LHC $(14 \mathrm{TeV})$. Here $\theta_{\ell^{+}}$is the polar angle of $\ell^{+}$in the $t$ rest frame with respect to the $t$ direction in the $t \bar{t}$ ZMF. The NLOW results are for $\mu=m_{t}$ (solid), $m_{t} / 2$ (dashed), and $2 m_{t}$ (dotted).

holds in the SM to a high degree of accuracy if the selection cuts/criteria are CP images of each other. Although the $p p$ state is not a CP eigenstate, one can show that (IV.19) holds as long as $g g$ and $q \bar{q}$ initiated $t \bar{t}$ production is governed by CP-invariant interactions [112].

One may use these distributions for checks of whether or not the collected ensemble of (anti)top quarks has a longitudinal polarization, which would be due to some new parity-violating interaction that affects $t \bar{t}$ production 11 . That is, one may investigate whether or not the measured distributions differ from the SM results, Fig. 20.

If one defines $\cos \theta_{\ell^{+}}=\hat{\mathbf{a}} \cdot \hat{\ell}^{+}$with respect to an axial vector $\hat{\mathbf{a}}$, e.g. the vector normal to the $2 \rightarrow 2$ scattering plane (and likewise for $\ell^{-}$), one may check for a normal polarization of top quarks generated by absorptive parts in the scattering amplitude. At NLO QCD this polarization amounts to a few percent [58,59].

We close this section with a few remarks on some distributions/correlations suitable for CP symmetry tests respectively searches for non-standard CP violation (cf., e.g., [109] for a review). This can be made with dileptonic and semileptonic $t \bar{t}$ events at the Tevatron and especially at the LHC, once sufficiently large data samples will have been collected. Although the (expectation values of the) observables below cannot be classified a priori with respect to $\mathrm{CP}$ in the case of $p p$ collisions, they may nevertheless be used at the LHC. As shown in [110,111] by a general kinematic analysis, $\mathrm{P}$ - and CP-violating interactions that affect hadronic $t \bar{t}$ production induce, in general, at the level

\footnotetext{
${ }^{11}$ Related parity-violating polarization observables and their size in some SM extensions were investigated in [108].
} 
of the $t \bar{t}$ intermediate states two types of spin correlations/asymmetries:

1. A difference in the longitudinal polarization of $t$ and $\bar{t}$ quarks. The most useful asymmetry corresponds to $\hat{\mathbf{k}} \cdot\left(\mathbf{S}_{t}-\mathbf{S}_{\bar{t}}\right)$ where $\hat{\mathbf{k}}$ is the direction of the $t$ quark in the $t \bar{t} \mathrm{ZMF}$. In the dileptonic or semileptonic $t \bar{t}$ events these correlations lead to a difference in the distributions $G_{\ell^{ \pm}}$. One may consider the ratio

$$
\Delta_{C P}^{\ell}(z)=\frac{G_{\ell^{+}}(z)-G_{\ell^{-}}(z)}{G_{\ell^{+}}(z)+G_{\ell^{-}}(z)}
$$

As shown above, $\Delta_{C P}^{\ell}=0$ to NLOW in the SM.

A non-zero $\Delta_{C P}^{\ell}$ requires, apart from $\mathrm{P}$ and $\mathrm{CP}$ violation, also an absorptive part in the scattering matrix. A non-zero polarization asymmetry $\hat{\mathbf{k}} \cdot\left(\mathbf{S}_{t}-\mathbf{S}_{\bar{t}}\right)$ amounts to a difference $N\left(t_{L} \bar{t}_{L}\right)-N\left(t_{R} \bar{t}_{R}\right) \neq 0$ of the number of $t \bar{t}$ with negative and positive helicity [113]. In the case of a CP-violating Higgs sector, in particular if heavy spin-zero states with undefined CP parity exist that are resonantly produced in the $t \bar{t}$ channel, then suitable asymmetries evaluated in suitable bins around the position of the resonance [96] may be as large as a few percent [112].

In the context of leptonic charge asymmetries discussed in Sect. IV.1 it is natural to consider

$$
A_{C P}^{\ell}(y)=\frac{N_{\ell^{+}}(y)-N_{\ell^{-}}(-y)}{N_{\ell^{+}}(y)+N_{\ell^{-}}(-y)},
$$

or its integrated version,

$$
A_{C P}^{\ell}=\frac{\int_{y>0} d y N_{\ell^{+}}(y)-\int_{y<0} d y N_{\ell^{-}}(y)}{\int_{y>0} d y N_{\ell^{+}}(y)+\int_{y<0} d y N_{\ell^{-}}(y)} .
$$

The asymmetries (IV.21) and (IV.22) correspond to a top-spin asymmetry with respect to the beam direction. They are rather insensitive to P- and CP-violating Higgs-boson effects. Larger effects would result from $s$-channel exchanges of new heavy $J \neq 0$ resonances with CP-violating couplings to top quarks. A possible difference in the transverse energies of $\ell^{+}$ and $\ell^{-}$, which derives also from these single spin asymmetries, is below the percent level for non-resonant CP-violating exchanges of Higgs bosons [113] or of squarks/gluinos [114].

2. P- and CP-odd/T-odd $t \bar{t}$ spin correlations. The most useful one is proportional to $\hat{\mathbf{k}} \cdot\left(\mathbf{S}_{t} \times \mathbf{S}_{\bar{t}}\right)$. It can be induced already at tree level. In dileptonic events it would show up in correlations proportional to $\mathcal{O}_{C P}=\hat{\mathbf{k}} \cdot\left(\hat{\ell}^{+} \times \hat{\ell}^{-}\right)$, and likewise for semileptonic events. When the unit momenta $\hat{\ell}^{+}\left(\hat{\ell}^{-}\right)$and $\hat{\mathbf{k}}$ are taken in the $t(\bar{t})$ rest frame and in the $t \bar{t} \mathrm{ZMF}$, respectively, then $\mathcal{O}_{C P}$ corresponds, in the absence of cuts, to a term proportional to $\sin \Delta \phi^{*}$, where $\Delta \phi^{*}$ denotes the difference of the $\ell^{+}, \ell^{-}$azimuthal angles in the plane orthogonal to $\hat{\mathbf{k}}$. As the construction of these frames with reasonably small experimental error will be difficult, one may use pseudo rest-frames in analogy to what was shown in [115].

In the case of resonant $s$-channel exchange of a Higgs particle with scalar and pseudoscalar 
Yukawa couplings, suitable asymmetries $A_{C P} \propto N\left(\mathcal{O}_{C P}>0\right)-N\left(\mathcal{O}_{C P}<0\right)$ may be as large as a few percent when evaluated in appropriate bins around the position of the resonance [96,112], and do not receive SM contributions at the few per mille level.

The above dileptonic azimuthal angle correlation in the laboratory frame, $\sigma^{-1} d \sigma / d \Delta \phi$, is symmetric with respect to $\Delta \phi \rightarrow 2 \pi-\Delta \phi$ in the case of CP invariance. CP-violating interactions would, in general, modulate it by a term proportional to $\sin \Delta \phi$ (in the absence of cuts); i.e. one should measure it in the whole range $0 \leq \Delta \phi<2 \pi$. In the terminology of [110] this term corresponds to a correlation of $\mathbf{S}_{t} \times \mathbf{S}_{\bar{t}}$ with the beam direction in the laboratory frame, i.e., to a correlation observable $\hat{\mathbf{p}}_{L} \cdot\left(\hat{\boldsymbol{\ell}}_{\boldsymbol{L}}^{+} \times \hat{\boldsymbol{\ell}}_{\boldsymbol{L}}^{-}\right)$. As far as possible Higgs sector $\mathrm{CP}$ violation is concerned, this correlation is rather insensitive to resonant terms if the mass of the resonance is significantly above the $t \bar{t}$ production threshold. The non-resonant Higgs-boson exchange terms lead to rather small CP-violating effects [111].

\section{Conclusions}

We have investigated a number of observables that are and will be instrumental in the exploration of $t \bar{t}$ production and decay at the Tevatron and the LHC. For this analysis we made a computer program that incorporates besides the NLO QCD corrections to $t \bar{t}$ production and decay also mixed weak-QCD corrections to the production amplitudes, and that allows for studies of correlated versus uncorrelated $t \bar{t}$ events.

We assessed, for a few distributions and correlations at the LHC, the relative size of the weakinteraction contributions as compared to the NLO QCD results. In the $p_{T}$ or $M_{t \bar{t}}$ range where most of the $t \bar{t}$ events at the LHC are located, these contributions deplete the NLO QCD results somewhat, for instance, the $p_{T}$ and $M_{t \bar{t}}$ distribution by about $-2 \%$ for $p_{T} \sim 400 \mathrm{GeV}$ and $M_{t \bar{t}} \sim 1.2$ $\mathrm{TeV}$. For larger $p_{T}, M_{t \bar{t}}$ the depletion grows to several percent. As to the charge asymmetries $A$ and $A^{t \bar{t}}$ computed at the level of intermediate $t \bar{t}$ : our results agree with [45]; yet we find that the weak-interaction contributions are slightly smaller than those determined in [43], due to additional terms that were previously not taken into account.

Our main physics results are the investigations at NLOW in the gauge couplings of the asymmetries and angular correlations for dileptonic events which were presented in Sect. IV. First, we considered charge asymmetries at the Tevatron. The pair-asymmetry $A^{t \bar{t}}$ decreases by about $10 \%$ if acceptance cuts are applied. Our result, $A^{t \bar{t}}=0.071(7)$ (where the given uncertainty due to scale variations certainly underestimates the true theoretical error), may be compared with the respective D0 measurement [47]. Both results agree within the rather large uncertainties. We computed also a leptonic charge asymmetry $A^{\ell}$ and a pair-asymmetry $A^{\ell \ell}$ for the Tevatron, which are related to $A$ and $A^{t \bar{t}}$, respectively. As in the case of $A^{t \bar{t}}$, we determined these asymmetries for correlated and uncorrelated $t \bar{t}$ events, in order to study the effect of spin correlations on these distributions. We showed that $t \bar{t}$ spin correlations affect the pair asymmetry $A^{\ell \ell}$ by about $7 \%$ for the acceptance cuts used in Sect. IV. These leptonic asymmetries have, to our knowledge, not yet been measured, probably because of limited numbers of (di)leptonic events. As these leptonic 
asymmetries should eventually be measurable more precisely than $A$ or $A^{t \bar{t}}$, their experimental determination might provide a more conclusive comparison with SM results. Our NLOW predictions are given in Table 3. One may speculate that top-spin effects are more pronounced in more exclusive charge or forward-backward asymmetries [44], but this remains to be investigated.

Furthermore, we determined several dileptonic angular correlations, which reflect $t \bar{t}$ spin correlations with respect to different spin bases, namely the beam, off-diagonal and helicity correlation, and an opening angle distribution defined in a specific way [14, when selection cuts are applied. Our NLOW predictions for the beam, off-diagonal, and helicity correlation for the Tevatron agree with recent measurements by the CDF and D0 experiments; albeit the experimental uncertainties are still large, mainly because of limited statistics.

We have also made predictions for estimators (which have 1-dimensional distributions) of these correlations as functions of $M_{t \bar{t}}$. These estimators may prove useful for the Tevatron and also in the early rounds of LHC data-analyses, where the event numbers will likely not be abundant.

We have also considered two angular correlations defined in the laboratory frame for dileptonic final states at the LHC, and we have computed them to NLOW for correlated and uncorrelated $t \bar{t}$ events. Our results confirm the findings of [64], namely that the azimuthal angle correlation is a sensitive variable for exploring $t \bar{t}$ spin correlations at the LHC in the low-energy tail of the $M_{t \bar{t}}$ spectrum $\left(M_{t \bar{t}}<400 \mathrm{GeV}\right)$. It should be recalled that the helicity correlation and the opening angle distribution as defined in [14] are sensitive to spin correlations also when applied to event samples with $M_{t \bar{t}}^{\text {cut }}>400 \mathrm{GeV}$. An crucial experimental issue in the precise measurement of both the azimuthal angle correlation and the latter observables is the kinematic reconstruction of the dileptonic events.

Moreover, we have briefly discussed a few P- and CP-odd observables that should be useful in the search for $\mathrm{P}$ - and/or CP-violating interactions in dileptonic and semileptonic $t \bar{t}$ events. Our NLOW results on distributions and correlations indicate that the above observables do not receive SM contributions at the level of a few per mille or below. This issue deserves further, more detailed studies.

It is also worth recalling here that, in case the measurements of the $t \bar{t}$ spin correlations at the LHC should eventually match the SM predictions - for instance predictions that involve the low-energy tail of the $M_{t \bar{t}}$ spectrum - and would reach a reasonable level of precision, one may use these correlations also for the further exploration of the parton content of the proton. They are, in fact, quite sensitive to the proton's relative quark and gluon content, because the contributions from $g g$ and $q \bar{q}$ initiated $t \bar{t}$ production have opposite signs [13, 14].

The above investigations and comparisons with existing experimental results show that the precision, with which some distributions, especially angular correlations can be predicted, will likely not be matched quickly by experiments in the near future. Nevertheless, in view of the potential importance of observables such as the leptonic charge asymmetries and spin correlations in exploring the interactions of top quarks, further detailed investigations on expected measurement uncertainties at the LHC would certainly not be futile exercises. 
Future extensions of our work may include the implementation of threshold resummations into our code and, as to more phenomenological issues, an analysis of charge asymmetries and angular correlations at NLOW also for lepton + jets final states, and a more systematic error analysis by taking into account also other available PDF sets [116, 117] including the PDF uncertainties.

\section{Acknowledgements}

We thank Jorgen D'Hondt, Eric Laenen, Christian Schwanenberger, Peter Uwer and Wolfgang Wagner for discussions and information about their work. Z.G. Si wishes to thank RWTH Aachen for its hospitality during a stay where this work was completed, and the Deutsche Forschungsgemeinschaft (DFG) and the Ministry of Education (China) for an exchange grant. His work was supported in part by NSFC and by Natural Science Foundation of Shandong Province and that of W.B. by DFG, SFB TR9. 


\section{References}

[1] P. Nason, S. Dawson and R. K. Ellis, Nucl. Phys. B 303 (1988) 607.

[2] P. Nason, S. Dawson and R. K. Ellis, Nucl. Phys. B 327 (1989) 49.

[3] W. Beenakker, H. Kuijf, W. L. van Neerven and J. Smith, Phys. Rev. D 40 (1989) 54.

[4] W. Beenakker, W. L. van Neerven, R. Meng, G. A. Schuler and J. Smith, Nucl. Phys. B 351 (1991) 507.

[5] M. L. Mangano, P. Nason and G. Ridolfi, Nucl. Phys. B 373 (1992) 295.

[6] S. Frixione, M. L. Mangano, P. Nason and G. Ridolfi, Phys. Lett. B 351 (1995) 555 arXiv:hep-ph/9503213.

[7] E. Laenen, J. Smith and W. L. van Neerven, Phys. Lett. B 321 (1994) 254 arXiv:hep-ph/9310233.

[8] N. Kidonakis and G. Sterman, Nucl. Phys. B 505 (1997) 321 arXiv:hep-ph/9705234.

[9] R. Bonciani, S. Catani, M. L. Mangano and P. Nason, Nucl. Phys. B 529 (1998) 424 [Erratumibid. B 803 (2008) 234] arXiv:hep-ph/9801375].

[10] N. Kidonakis, E. Laenen, S. Moch and R. Vogt, Phys. Rev. D 64 (2001) 114001 arXiv:hep-ph/0105041.

[11] W. Bernreuther, A. Brandenburg and Z. G. Si, Phys. Lett. B 483 (2000) 99 arXiv:hep-ph/0004184.

[12] W. Bernreuther, A. Brandenburg, Z. G. Si and P. Uwer, Phys. Lett. B 509 (2001) 53 arXiv:hep-ph/0104096.

[13] W. Bernreuther, A. Brandenburg, Z. G. Si and P. Uwer, Phys. Rev. Lett. 87 (2001) 242002 arXiv:hep-ph/0107086].

[14] W. Bernreuther, A. Brandenburg, Z. G. Si and P. Uwer, Nucl. Phys. B 690 (2004) 81 arXiv:hep-ph/0403035.

[15] S. Moch and P. Uwer, Phys. Rev. D 78 (2008) 034003 arXiv:0804.1476 [hep-ph]].

[16] M. Cacciari, S. Frixione, M. L. Mangano, P. Nason and G. Ridolfi, JHEP 0809 (2008) 127 arXiv:0804.2800 [hep-ph]].

[17] N. Kidonakis and R. Vogt, Phys. Rev. D 78 (2008) 074005 [arXiv:0805.3844 [hep-ph]].

[18] W. Beenakker, A. Denner, W. Hollik, R. Mertig, T. Sack and D. Wackeroth, Nucl. Phys. B 411 (1994) 343. 
[19] W. Bernreuther, M. Fuecker and Z. G. Si, Phys. Lett. B 633 (2006) 54 arXiv:hep-ph/0508091.

[20] W. Bernreuther, M. Fuecker and Z. G. Si, Phys. Rev. D 74 (2006) 113005 arXiv:hep-ph/0610334.

[21] W. Bernreuther, M. Fücker and Z. G. Si, Phys. Rev. D 78 (2008) 017503 arXiv:0804.1237 [hep-ph]].

[22] J. H. Kühn, A. Scharf and P. Uwer, Eur. Phys. J. C 45 (2006) 139 arXiv:hep-ph/0508092].

[23] J. H. Kühn, A. Scharf and P. Uwer, Eur. Phys. J. C 51 (2007) 37 arXiv:hep-ph/0610335.

[24] S. Moretti, M. R. Nolten and D. A. Ross, Phys. Lett. B 639 (2006) 513 [Erratum-ibid. B 660 (2008) 607] arXiv:hep-ph/0603083.

[25] W. Hollik and M. Kollar, Phys. Rev. D 77 (2008) 014008 arXiv:0708.1697 [hep-ph]].

[26] W. Beenakker, F. A. Berends and A. P. Chapovsky, Phys. Lett. B 454 (1999) 129 arXiv:hep-ph/9902304.

[27] S. Dittmaier, P. Uwer and S. Weinzierl, Phys. Rev. Lett. 98 (2007) 262002 arXiv:hep-ph/0703120.

[28] S. Dittmaier, P. Uwer and S. Weinzierl, Eur. Phys. J. C 59 (2009) 625 arXiv:0810.0452 [hep-ph]].

[29] A. Bredenstein, A. Denner, S. Dittmaier and S. Pozzorini, Phys. Rev. Lett. 103 (2009) 012002 arXiv:0905.0110 [hep-ph]].

[30] A. Bredenstein, A. Denner, S. Dittmaier and S. Pozzorini, arXiv:1001.4006 [hep-ph].

[31] G. Bevilacqua, M. Czakon, C. G. Papadopoulos, R. Pittau and M. Worek, JHEP 0909 (2009) 109 arXiv:0907.4723 [hep-ph]].

[32] G. Bevilacqua, M. Czakon, C. G. Papadopoulos and M. Worek, arXiv:1002.4009 [hep-ph].

[33] A. Czarnecki, M. Jezabek and J. H. Kühn, Nucl. Phys. B 351 (1991) 70.

[34] A. Brandenburg, Z. G. Si and P. Uwer, Phys. Lett. B 539 (2002) 235 arXiv:hep-ph/0205023.

[35] A. Czarnecki and K. Melnikov, Nucl. Phys. B 544 (1999) 520 arXiv:hep-ph/9806244.

[36] K. G. Chetyrkin, R. Harlander, T. Seidensticker and M. Steinhauser, Phys. Rev. D 60 (1999) 114015 arXiv:hep-ph/9906273.

[37] W. Bernreuther, J. Phys. G 35 (2008) 083001 [arXiv:0805.1333 [hep-ph]].

[38] J. R. Incandela, A. Quadt, W. Wagner and D. Wicke, Prog. Part. Nucl. Phys. 63 (2009) 239 arXiv:0904.2499 [hep-ex]]. 
[39] C. Schwanenberger, plenary talk at EPS HEP conference, Krakow (2009).

[40] H. S. Do, S. Groote, J. G. Körner and M. C. Mauser, Phys. Rev. D 67 (2003) 091501 arXiv:hep-ph/0209185|.

[41] J. H. Piclum, A. Czarnecki and J. G. Körner, Nucl. Phys. Proc. Suppl. 183 (2008) 48.

[42] F. Halzen, P. Hoyer and C. S. Kim, Phys. Lett. B 195 (1987) 74.

[43] J. H. Kühn and G. Rodrigo, Phys. Rev. D 59 (1999) 054017 [arXiv:hep-ph/9807420].

[44] M. T. Bowen, S. D. Ellis and D. Rainwater, Phys. Rev. D 73 (2006) 014008 arXiv:hep-ph/0509267.

[45] O. Antunano, J. H. Kühn and G. V. Rodrigo, Phys. Rev. D 77 (2008) 014003 arXiv:0709.1652 [hep-ph]].

[46] L. G. Almeida, G. Sterman and W. Vogelsang, Phys. Rev. D 78 (2008) 014008 [arXiv:0805.1885 [hep-ph]].

[47] V. M. Abazov et al. [D0 Collaboration], Phys. Rev. Lett. 100 (2008) 142002 arXiv:0712.0851 [hep-ex]].

[48] T. Aaltonen et al. [CDF Collaboration], Phys. Rev. Lett. 101 (2008) 202001 arXiv:0806.2472 [hep-ex]].

[49] CDF collaboration, public note CDF/ANAL/TOP/PUBLIC/9724 (2009).

[50] J. H. Kühn, Nucl. Phys. B 237 (1984) 77.

[51] V. D. Barger, J. Ohnemus and R. J. Phillips, Int. J. Mod. Phys. A 4 (1989) 617.

[52] G. L. Kane, G. A. Ladinsky and C. P. Yuan, Phys. Rev. D 45 (1992) 124.

[53] T. Arens and L. M. Sehgal, Phys. Lett. B 302 (1993) 501.

[54] G. Mahlon and S. Parke, Phys. Rev. D 53 (1996) 4886 arXiv:hep-ph/9512264].

[55] T. Stelzer and S. Willenbrock, Phys. Lett. B 374 (1996) 169 arXiv:hep-ph/9512292].

[56] A. Brandenburg, Phys. Lett. B 388 (1996) 626 arXiv:hep-ph/9603333].

[57] D. Chang, S. C. Lee and A. Sumarokov, Phys. Rev. Lett. 77 (1996) 1218 arXiv:hep-ph/9512417.

[58] W. Bernreuther, A. Brandenburg and P. Uwer, Phys. Lett. B 368 (1996) 153 arXiv:hep-ph/9510300].

[59] W. G. Dharmaratna and G. R. Goldstein, Phys. Rev. D 53 (1996) 1073. 
[60] G. Mahlon and S. Parke, Phys. Lett. B 411 (1997) 173 arXiv:hep-ph/9706304.

[61] P. Uwer, Phys. Lett. B 609 (2005) 271 arXiv:hep-ph/0412097].

[62] C. A. Nelson, E. G. Barbagiovanni, J. J. Berger, E. K. Pueschel and J. R. Wickman, Eur. Phys. J. C 45 (2006) 121 [arXiv:hep-ph/0506240].

[63] R. M. Godbole, S. D. Rindani and R. K. Singh, JHEP 0612 (2006) 021 arXiv:hep-ph/0605100.

[64] G. Mahlon and S. J. Parke, arXiv:1001.3422 [hep-ph].

[65] D0 collaboration, Conference Note 5950-CONF (2009).

[66] CDF collaboration, CDF note 9824 (2009).

[67] CDF collaboration, CDF note 10048 (2010).

[68] U. Langenfeld, S. Moch and P. Uwer, Phys. Rev. D 80 (2009) 054009 arXiv:0906.5273 [hep$\mathrm{ph}]]$.

[69] M. Czakon and A. Mitov, Nucl. Phys. B 824 (2010) 111 [arXiv:0811.4119 [hep-ph]].

[70] M. Czakon and A. Mitov, Phys. Lett. B 680 (2009) 154 [arXiv:0812.0353 [hep-ph]].

[71] M. Czakon, A. Mitov and G. Sterman, Phys. Rev. D 80 (2009) 074017 arXiv:0907.1790 [hep-ph]].

[72] M. Beneke, P. Falgari and C. Schwinn, Nucl. Phys. B 828 (2010) 69 arXiv:0907.1443 [hep$\mathrm{ph}]$.

[73] M. Beneke, M. Czakon, P. Falgari, A. Mitov and C. Schwinn, arXiv:0911.5166 [hep-ph].

[74] V. Ahrens, A. Ferroglia, M. Neubert, B. D. Pecjak and L. L. Yang, arXiv:0912.3375 [hep-ph].

[75] K. Hagiwara, Y. Sumino and H. Yokoya, Phys. Lett. B 666 (2008) 71 arXiv:0804.1014 [hep$\mathrm{ph}]$.

[76] Y. Kiyo, J. H. Kühn, S. Moch, M. Steinhauser and P. Uwer, Eur. Phys. J. C 60 (2009) 375 arXiv:0812.0919 [hep-ph]].

[77] M. Czakon, A. Mitov and S. Moch, Nucl. Phys. B 798 (2008) 210 [arXiv:0707.4139 [hep-ph]].

[78] M. Czakon, Phys. Lett. B 664 (2008) 307 [arXiv:0803.1400 [hep-ph]].

[79] B. Kniehl, Z. Merebashvili, J. G. Körner and M. Rogal, Phys. Rev. D 78 (2008) 094013 arXiv:0809.3980 [hep-ph]].

[80] C. Anastasiou and S. M. Aybat, Phys. Rev. D 78 (2008) 114006 arXiv:0809.1355 [hep-ph]]. 
[81] R. Bonciani, A. Ferroglia, T. Gehrmann and C. Studerus, JHEP 0908 (2009) 067 arXiv:0906.3671 [hep-ph]].

[82] T. Becher and M. Neubert, Phys. Rev. D 79 (2009) 125004 [Erratum-ibid. D 80 (2009) 109901] arXiv:0904.1021 [hep-ph]].

[83] A. Ferroglia, M. Neubert, B. D. Pecjak and L. L. Yang, JHEP 0911 (2009) 062 arXiv:0908.3676 [hep-ph]].

[84] D. E. Kaplan, K. Rehermann, M. D. Schwartz and B. Tweedie, Phys. Rev. Lett. 101 (2008) 142001 [arXiv:0806.0848 [hep-ph]].

[85] L. G. Almeida, S. J. Lee, G. Perez, I. Sung and J. Virzi, Phys. Rev. D 79 (2009) 074012 arXiv:0810.0934 [hep-ph]].

[86] P. M. Nadolsky et al., Phys. Rev. D 78 (2008) 013004 [arXiv:0802.0007 [hep-ph]].

[87] S. Frixione and B. R. Webber, arXiv:0812.0770 [hep-ph].

[88] S. Frixione, E. Laenen, P. Motylinski and B. R. Webber, JHEP 0704 (2007) 081 [arXiv:hep-ph/0702198].

[89] J. Campbell and R. K. Ellis, http:/mcfm.fnal.gov

[90] S. Frixione, P. Nason and G. Ridolfi, JHEP 0709 (2007) 126 [arXiv:0707.3088 [hep-ph]].

[91] K. Melnikov and M. Schulze, JHEP 0908 (2009) 049 [arXiv:0907.3090 [hep-ph]].

[92] [Tevatron Electroweak Working Group and CDF Collaboration and D0 Collab], arXiv:0903.2503 [hep-ex].

[93] W. Bernreuther and W. Wetzel, Nucl. Phys. B 197 (1982) 228 [Erratum-ibid. B 513 (1998) $758]$.

[94] C. Amsler et al. [Particle Data Group], Phys. Lett. B 667 (2008) 1.

[95] S. Catani, Y. L. Dokshitzer and B. R. Webber, Phys. Lett. B 285 (1992) 291.

[96] W. Bernreuther, M. Flesch and P. Haberl, Phys. Rev. D 58 (1998) 114031 arXiv:hep-ph/9709284.

[97] D. Dicus, A. Stange and S. Willenbrock, Phys. Lett. B 333 (1994) 126 arXiv:hep-ph/9404359.

[98] V. Barger, T. Han and D. G. E. Walker, Phys. Rev. Lett. 100 (2008) 031801 arXiv:hep-ph/0612016.

[99] K. Agashe et al., Phys. Rev. D 76 (2007) 115015 [arXiv:0709.0007 [hep-ph]]. 
[100] U. Baur and L. H. Orr, Phys. Rev. D 77 (2008) 114001 arXiv:0803.1160 [hep-ph]].

[101] R. Frederix and F. Maltoni, JHEP 0901 (2009) 047 [arXiv:0712.2355 [hep-ph]].

[102] L. M. Sehgal and M. Wanninger, Phys. Lett. B 200 (1988) 211.

[103] W. Bernreuther, P. Gonzalez and M. Wiebusch, Eur. Phys. J. C 60 (2009) 197 arXiv:0812.1643 [hep-ph]].

[104] J. D' Hondt, private communication.

[105] L. Sonnenschein, Phys. Rev. D 72 (2005) 095020 arXiv:hep-ph/0510100.

[106] F. Hubaut, E. Monnier, P. Pralavorio, K. Smolek and V. Simak, Eur. Phys. J. C 44S2 (2005) 13 arXiv:hep-ex/0508061.

[107] W. Bernreuther and Z. G. Si, in preparation.

[108] C. Kao and D. Wackeroth, Phys. Rev. D 61 (2000) 055009 arXiv:hep-ph/9902202.

[109] D. Atwood, S. Bar-Shalom, G. Eilam and A. Soni, Phys. Rept. 347 (2001) 1 arXiv:hep-ph/0006032.

[110] W. Bernreuther and A. Brandenburg, Phys. Lett. B 314 (1993) 104.

[111] W. Bernreuther and A. Brandenburg, Phys. Rev. D 49 (1994) 4481 [arXiv:hep-ph/9312210].

[112] W. Bernreuther, A. Brandenburg and M. Flesch, arXiv:hep-ph/9812387.

[113] C. R. Schmidt and M. E. Peskin, Phys. Rev. Lett. 69 (1992) 410.

[114] C. R. Schmidt, Phys. Lett. B 293 (1992) 111.

[115] S. Berge and W. Bernreuther, Phys. Lett. B 671 (2009) 470 [arXiv:0812.1910 [hep-ph]].

[116] A. D. Martin, W. J. Stirling, R. S. Thorne and G. Watt, Eur. Phys. J. C 63 (2009) 189 arXiv:0901.0002 [hep-ph]].

[117] S. Alekhin, J. Blümlein, S. Klein and S. Moch, arXiv:0908.2766 [hep-ph]. 